\title{
Divergence and Gene Flow Between Fusarium subglutinans and F. temperatum Isolated from Maize in Argentina
}

\author{
M. Veronica Fumero, ${ }^{1}$ Wei Yue, ${ }^{2}$ María L. Chiotta, ${ }^{1}$ Sofía N. Chulze, ${ }^{1}$ John F. Leslie, ${ }^{2}$ and Christopher Toomajian ${ }^{2, \dagger}$ \\ ${ }^{1}$ Research Institute on Mycology and Mycotoxicology (IMICO), National Scientific and Technical Research Council-National University of Río \\ Cuarto (CONICET-UNRC), X5800, Río Cuarto, Córdoba, Argentina \\ ${ }^{2}$ Department of Plant Pathology, Kansas State University, Manhattan, KS 66506, U.S.A. \\ Accepted for publication 15 October 2020.
}

\begin{abstract}
Fusarium subglutinans and F. temperatum are two important fungal pathogens of maize whose distinctness as separate species has been difficult to assess. We isolated strains of these species from commercial and native maize varieties in Argentina and sequenced $>28,000$ loci to estimate genetic variation in the sample. Our objectives were to measure genetic divergence between the species, infer demographic parameters related to their split, and describe the population structure of the sample. When analyzed together, over $30 \%$ of each species' polymorphic sites ( $>2,500$ sites) segregate as polymorphisms in the other. Demographic modeling confirmed the species split predated maize domestication, but subsequent between-species gene flow has occurred, with gene flow from $F$. subglutinans into $F$. temperatum greater than gene flow in the reverse direction. In $F$. subglutinans, little evidence exists for substructure or recent selective sweeps, but there is evidence for limited sexual reproduction. In F. temperatum, there is clear evidence for population
\end{abstract}

ABSTRACT substructure and signals of abundant recent selective sweeps, with sexual reproduction probably less common than in F. subglutinans. Both genetic variation and the relative number of polymorphisms shared between species increase near the telomeres of all 12 chromosomes, where genes related to plant-pathogen interactions often are located. Our results suggest that species boundaries between closely related Fusarium species can be semipermeable and merit further study. Such semipermeability could facilitate unanticipated genetic exchange between species and enable quicker permanent responses to changes in the agro-ecosystem, e.g., pathogen-resistant host varieties, new chemical and biological control agents, and agronomic practices.

Keywords: fungal pathogens, Fusarium subglutinans, Fusarium temperatum, GBS markers, gene flow, genomics, genetics, population biology, population structure, species boundary
The genus Fusarium of filamentous ascomycete fungi comprises numerous species distributed worldwide, many of which are toxigenic plant pathogens that cause economic losses due to reductions in quantity and quality of staple foods (Leslie and Summerell 2006). Within the genus, there is a wide range of infection and reproductive strategies, and variation in the degree of host specialization. Fusarium species are grouped in species

${ }^{\dagger}$ Corresponding author: C. Toomajian; toomajia@ksu.edu

Funding: This work was supported in part by the United States Agency for International Development (USAID) Feed the Future Innovation Lab for the Reduction of Post-Harvest Loss (PHLIL) (Cooperative Agreement AID-OAA-L14-00004), the National Institute of Food and Agriculture Kansas Agricultural Experiment Station Hatch Multi/state project KS1183A, and the USDA Agriculture Research Service U.S. Wheat \& Barley Scab Initiative cooperative project under Agreement number 59-0206-1-113. Any opinions, findings, conclusions, or recommendations expressed in this publication are those of the authors and do not necessarily reflect the view of the U.S. Department of Agriculture, USAID, or the U.S. government. Portions of the computing for this project was performed on the Beocat Research Cluster at Kansas State University, which is funded in part by National Science Foundation grants ACI-1440548, CHE-1726332, CNS-1006860, EPS-1006860, EPS-0919443, and National Institute of General Medical Sciences P20GM113109. M. V. Fumero's travel and research at Kansas State University was supported by the 2015 BEC.AR-Fulbright Program of the Ministry of Education of Argentina. Portions of this work were based on studies described in M. V. Fumero's Ph.D. thesis at the National University of Río Cuarto (Río Cuarto, Córdoba, Argentina), and in W. Yue's Ph.D. thesis at Kansas State University (Manhattan, $\mathrm{KS})$. This is contribution number 20-070-J from the Kansas Agricultural Experiment Station.

*The $e$-Xtra logo stands for "electronic extra" and indicates there are supplementary materials and supplementary tables published online.

The author(s) declare no conflict of interest.

C 2021 The American Phytopathological Society complexes (Aoki et al. 2014; Kvas et al. 2009; O'Donnell et al. 2013), of which the F. fujikuroi species complex (FFSC) is one of the largest. This species complex currently comprises 65 species that infect a wide range of native and cultivated plants. Some species specialize on a single primary host, while others have a broader host range (Al-Hatmi et al. 2016; Edwards et al. 2016; Herron et al. 2015; Laurence et al. 2015; Moussa et al. 2017; O’Donnell et al. 2015).

Like many of the Fusarium species complexes, the FFSC has a history of major expansion, as species defined initially on morphology have been reevaluated with other criteria. Focusing on species in this complex that are sexual and heterothallic, the biological species concept has been extensively employed to identify species (Leslie 1991), including F. subglutinans (mating population E), a maize pathogen found worldwide (Moretti et al. 1995). DNA analysis of strains identified as F. subglutinans sensu lato recognized multiple cryptic lineages, two of which are now described as the closely related species, F. subglutinans sensu stricto and F. temperatum (Scauflaire et al. 2011; Steenkamp et al. 2002). While molecular markers are consistent with separation into two well-supported clades, in sexual crosses under laboratory conditions a low level of interfertility exists between strains in the different species (Fumero et al. 2015; Levic et al. 2018).

Besides facilitating species identification, inexpensive DNA sequencing has, with the proliferation of tools for assembly and annotation, led to a major increase in genomic studies on Fusarium species in the last decade. Genome sequences are now available for numerous Fusarium species, including $F$. avenaceum (Lysøe et al. 2014), F. circinatum (Wingfield et al. 2018), F. fujikuroi (Wiemann et al. 2013), F. graminearum (King et al. 2017; Ma et al. 2010), F. solani (Coleman et al. 2009), F. udum (Srivastava et al. 2018), F. venenatum (King et al. 2018), F. verticillioides (Gardiner 2018; Ma et al. 2010), F. virguliforme (Srivastava et al. 2014), and many formae speciales of F. oxysporum (Asai et al. 2019; Guo et al. 2014; 
Seo et al. 2020). Evidence for genome compartmentalization and horizontal gene transfer have been major findings in many of these studies, with a major role for supernumerary chromosomes in both genome compartmentalization and horizontal gene transfer in F. oxysporum and F. solani (Coleman et al. 2009; Zhang and Ma 2017).

The first genomic study in Fusarium used crossovers from a genetic map and a comparison of two strains to identify segments of the $F$. graminearum genome, including subtelomeric regions, characterized by much higher variation and recombination than the rest of the genome (Cuomo et al. 2007). An analysis of gene content and expression found these same regions enriched for secreted genes or genes expressed in planta (Cuomo et al. 2007). These findings now have been found to apply to a number of other fungi, both within and outside the genus Fusarium, as well (Bertazzoni et al. 2018).

The first major comparative genomics study found that the genomes of $F$. graminearum, $F$. verticillioides, and $F$. oxysporum have discrete core and adaptive regions, the latter determining host specificity and interaction (Ma et al. 2010). It also identified lineage specific, or supernumerary, chromosomes in $F$. oxysporum, which could be transferred between strains and enable pathogenicity on particular plant hosts (Ma et al. 2010). Additional studies used genomics to reveal the diversification, coordinated regulation, and occasional horizontal transfer of secondary metabolite biosynthetic gene clusters (Fumero et al. 2020a; Niehaus et al. 2016a, b; Tralamazza et al. 2019; Villani et al. 2019; Wiemann et al. 2013). The public availability of these well-annotated genomes enabled the identification of patterns of purifying and site-specific diversifying selection contrasted between the core and adaptive regions of Fusarium genomes (Sperschneider et al. 2015).

More attention is now being given to investigating within-species variation at the genome scale. Many studies compare a few genomes of a particular species to evaluate variation in gene content (Walkowiak et al. 2015) or regulation (Guo et al. 2016; Kumar et al. 2010), especially for genes related to secondary metabolism. Similar studies have evaluated structural variation (Chiara et al. 2015) or the enrichment of genetic variants in genomic islands of high polymorphism (Laurent et al. 2017). The first true population genomic studies employed strains of $F$. graminearum. Sequencing restriction site-associated DNA fragments (RADseq) from hundreds of field isolates enabled studies of population structure and recombination hotspots (Talas and McDonald 2015). Resequencing whole genomes on a population scale remains challenging, but resequencing strategic isolates has helped to define the $F$. graminearum pan-genome and to highlight genome regions and sets of genes most likely involved in the differentiation of the species' main populations in the United States (Kelly and Ward 2018).

The objective of this study was to explore the patterns of genetic divergence between $F$. subglutinans and $F$. temperatum using a genotyping by sequencing (GBS) protocol to make evolutionary and demographic inferences about their split. Secondary aims included comparing the species in terms of levels of genetic diversity and evidence for population structure, genetic recombination, and recent selective sweeps. The data in this study were used to estimate when $F$. subglutinans and $F$. temperatum split into distinct units and also support a history of gene flow between the two during the process of speciation. The rate of gene flow from $F$. subglutinans into $F$. temperatum was higher than that from $F$. temperatum into $F$. subglutinans. Enrichment for shared polymorphisms between these species at most of the chromosome ends motivates interesting questions about the interaction between natural selection and gene flow during the divergence of these species. Pathogen population genomic investigations, by improving our understanding of pathogen biology and their evolution, can ultimately help with disease management and anticipating new disease outbreaks (Grünwald et al. 2016). Our study moves beyond comparative genomics of a few strains and provides an example of a bridge between an investigation of demographic processes within a species and comparative population genomics. This bridge enables us to outline potential processes involved in the speciation and divergence of two closely related sympatric species common to the same host with no demonstrated ecological niche differentiation.

\section{MATERIALS AND METHODS}

Field sampling. Maize samples were collected from two regions in Argentina where both $F$. subglutinans and $F$. temperatum had previously been recovered (Fumero et al. 2015): Humahuaca, Jujuy province (referred to in this study as NOA1) and Lajitas, Salta province. Maize samples also were collected from three other regions in Argentina: the southeast of Buenos Aires province (SEBA) because $F$. subglutinans had previously been found there (Reynoso et al. 2004), and Rapelli, Santiago de Estero province and Tartagal, Salta province to evaluate other locations near Lajitas. The different regions differ in maize varieties grown and span a gradient of values for elevation, mean annual temperature, and mean annual precipitation. No gross disease symptoms were detected in fields in SEBA, Lajitas, and Rapelli (all planted to commercial maize hybrids), but Fusarium was recovered from grain from all locations. Maize ears collected in fields from NOA1 (planted to unimproved local maize landraces; Cárcamo et al. 2011) and Tartagal (planted to a local white maize variety, 'Perla') usually were symptomatic, with pink-white mycelia visible at the ear tips. A more detailed description of the field sampling is provided as an online supplement (Supplementary Note S1).

Strain isolation and species identification. Strains of Fusarium were isolated from maize grains, subcultured, and identified morphologically following standard protocols (Leslie and Summerell 2006). Sexual crosses were made on carrot agar to determine biological species and male/female fertility as described by Leslie and Summerell (2006). Additional details on the strain isolation, morphological identification, and sexual crosses are provided in Supplementary Note S1.

Culture media. The media used in this study - peptone PCNB agar (PPA), Spezieller N"ahrstoffarmer agar (SNA), potato dextrose agar (PDA), carnation leaf agar (CLA), carrot agar (CA), and complete medium (CM) - were prepared according to Leslie and Summerell (2006).

DNA isolation, EF-1 $\alpha$ gene sequencing, and MAT PCR. Care was taken to extract high molecular weight genomic DNA from sample mycelia (Supplementary Note S2). We used a publicly available $E F-1 \alpha$ alignment containing data from 11 strains of $F$. subglutinans and 18 strains of $F$. temperatum (NCBI PopSet 756179050) to identify sites potentially diagnostic for each species (Fumero et al. 2015). Between positions 51 and 531 of the alignment, 11 of the polymorphic sites are fixed differences between the species. We sequenced a portion of the $E F-1 \alpha$ gene from the new strains included in this study (Supplementary Note $\mathrm{S} 2$ ). All of these strains matched either the $F$. subglutinans-specific or the F. temperatum-specific 11-site haplotype, and strains were assigned to species based on this haplotype. The MAT allele in each field isolate was determined following PCR amplification with selective primers (Steenkamp et al. 2000).

Genotyping by sequencing (GBS). A two-enzyme GBS protocol, modified from Poland et al. (2012), was performed in a 96 well plate that included strains of Fusarium and a negative control with water instead of DNA (Supplementary Note S2). The subset of strains included in this plate for analysis were a subset of larger sets that were initially collected and characterized. They are broadly representative of the sampling locations and MAT types, with preference for including strains with unusual toxigenic profiles (Fumero et al. 2015) or interspecies fertility phenotypes (Supplementary Table S1), when that information was available. For example, most strains of $F$. subglutinans produce both 
fusaproliferin and moniliformin and most strains of $F$. temperatum produce beauvericin, fusaproliferin and moniliformin, but preference was given to strains unable to produce one or more of the mycotoxins commonly produced in each species. The resulting library was sequenced with a HiSeq instrument (Illumina Inc., San Diego, CA) with a 100-cycle single read Illumina kit at the Genome Sequencing Facility of the University of Kansas Medical Center (Kansas City, KS).

GBS single nucleotide polymorphism (SNP) identification. Fastq files from the HiSeq output were separated into strains based on the barcodes of the GBS adapters by the software Geneious 7.1.7 (https://www.geneious.com). A sequential set of tools from Galaxy (Afgan et al. 2016) was used to trim reads in the Fastq files (Supplementary Note S3). BAM files were generated by aligning the trimmed and filtered reads to the $F$. temperatum CMWF389 genome (NCBI accession number PRJNA242703), with Bowtie2 software v. 2.1.0 using default settings in the Galaxy environment. The CMWF389 genome has a total length of 45,458,781 bp and is assembled in 43 scaffolds (Wingfield et al. 2015).

To construct putative chromosomes of $F$. temperatum and $F$. subglutinans, the $F$. temperatum reference genome scaffolds were compared with reference genomes of $F$. fujikuroi (GenBank assembly accession GCA_900079805.1) and F. verticillioides (GenBank-Genome accession number AAIM00000000.2) with the NUCmer pipeline of the MUMmer 3.0 software package (Kurtz et al. 2004). Approximate centromere positions were inferred by assuming orthologous locations with the $F$. fujikuroi genome (Wiemann et al. 2013), and the probable conformation of the resulting 12 chromosomes determined (Supplementary Table S2).

BAM files were analyzed with the Genome Analysis Toolkit (GATK v. 3.1-1) (McKenna et al. 2010) to identify high quality SNPs; and with STACKS software (version 1.04) (Catchen et al. 2013) to identify haplotypes from each strain at each GBS locus (Supplementary Note S3). As both species are haploid and thus should never be heterozygous at single-copy loci, traces of heterozygosity in STACKS haplotype calls were used to assess potential sample contamination. Evidence of heterozygosity from individual strains was assessed by identifying loci within strains with multiple haplotypes.

SNP mismatch distribution. A custom Perl script (Fumero et al. 2020b) was used to compare all pairs of strains at the 22,817 biallelic SNPs and summarize the proportion of SNPs that differed for each pair, either within or between species. Based on these pairwise comparisons, and large gaps in the distributions of pairwise differences observed within species (Fig. 1B), groups of closely related strains, or clonal lineages, were identified. We clustered strains such that all pairs of strains within a cluster differed at less than $1.8 \%$ of the SNPs for which both strains had a genotype call ( $<250$ total differences across all SNPs). None of the strains within clonal lineages are identical because even the most closely related pair of strains differed at 87 SNPs. For convenience hereafter, we use the terms close relatives and clones interchangeably. A clone-corrected set of strains was created by dropping all strains from the cluster except the one with the fewest missing SNP genotype calls. Whether the full set of strains or the smaller clonecorrected set from $F$. subglutinans and/or F. temperatum is used is specified for each population analysis.

Population structure analyses. These analyses were conducted with both the full sample and the clone-corrected set. A principal component analysis (PCA) was conducted with the Smartpca program of EIGENSOFT version 5.0.2 (Patterson et al. 2006). Custom Perl scripts were used to create PCA input files by filtering out singleton SNPs and those with genotype calls in fewer than half the strains of a given sample (Fumero et al. 2020b). The Bayesian clustering method implemented in STRUCTURE version 2.3.4 (Pritchard et al. 2000) was used to assign strains to subpopulations. STRUCTURE analyses included 20 independent runs for each potential value of $K$, where $K$, the number of groups, was varied from 1 to 20 . The fineRADstructure package (version 0 3_1; Malinsky et al. 2018) was used to further investigate the genetic structure of the populations, at both inter- and intraspecific levels. This software uses GBS-type data to infer recent shared ancestry in a sample and generates a coancestry matrix, i.e., a summary of nearest neighbor haplotype relationships. FineRADstructure utilizes the fineSTRUCTURE MCMC clustering algorithm (Lawson et al. 2012) to cluster individuals with recent shared ancestry based on the coancestry matrix, to infer the number of clusters, and to define the relationships between the clusters. Additional details on the population structure analyses are included as a supplement (Supplementary Note S4).

Linkage disequilibrium (LD) and recombination. The clone-corrected strain set was used for these analyses. After filtering SNPs based on levels of missing data (Supplementary Note S5), the $r^{2}$ and matrix options of PLINK software version 1.07 (Purcell et al. 2007) were used to compute $r^{2}$ for each SNP pair. A custom Perl script (Fumero et al. 2020b) was used to compute mean $r^{2}$ values for pairs of SNPs separated by 50 defined distance ranges (e.g., between 0 and $10 \mathrm{~kb}$, between 10 and $20 \mathrm{~kb}$, and up to between 490 and $500 \mathrm{~kb}$ ) or for SNPs from different scaffolds that were presumably unlinked. For each species, the midpoint between the mean $r^{2}$ value for SNPs separated by less than $10 \mathrm{~kb}$ (i.e., the maximum) and the mean for the unlinked SNPs (i.e., the minimum) was determined and used to estimate the physical distance at which the mean $r^{2}$ value has reached this midpoint. Pearson's correlation coefficient and Spearman's rank correlation coefficient between $r^{2}$ and the distance between SNPs (for SNPs $<500 \mathrm{~kb}$ apart) were calculated for both species. Following the procedure of Piganeau and Eyre-Walker (2004), we assessed the significance of the relationship between LD and distance in each species by performing a Mantel test (Sokal and Rohlf 1995; Supplementary Note S5).

The software RecMin (Myers and Griffiths 2003) was used to calculate the lower bound on the number of recombination events required to construct the history of our clone-corrected samples. The software computed the statistics $\mathrm{R}_{\mathrm{m}}$ (Hudson and Kaplan 1985), corresponding to the minimal number of different positions at which recombination occurred, and $\mathrm{R}_{\mathrm{h}}$ (Myers and Griffiths 2003), the minimum number of recombination events that have happened (as more than one event can occur at a position in the history of the sample). The same SNP sets as used for the $r^{2}$ analysis were used with parameters maximum subset size (-s) 10 and maximum width (-w) 20, but without using information on ancestral states.

Demographic analysis. The $\delta a \delta i$ package (version 1.7.0) (Gutenkunst et al. 2009) was used to infer the demographic history of F. subglutinans and F. temperatum. Demographic inferences made by $\delta a \delta i$ are based on the allele frequency spectrum and are more powerful when the ancestral and derived state of each SNP is known. The ancestral state of each SNP was inferred (Supplementary Note S6) by blasting 101 bp target sequences centered on each SNP against two outgroup genomes, i.e., F. verticillioides (GenBank-Genome accession number AAIM00000000.2) and F. circinatum (GenBankGenome accession number AYJV00000000.2).

The demographic inference of $\delta a \delta i$ requires SNPs with no missing data, but most of the GBS SNPs had some missing data. We found that the total number of SNPs available for the analysis was maximized when we projected down from the original sample size of 46 strains of $F$. subglutinans and 27 strains of $F$. temperatum to 9 and 5 strains, respectively, and thus used this projection for our analyses (Supplementary Note S6).

We tested for evidence of population size change within each species separately by comparing three single population (1D) models (growth, two epoch, and three epoch) with the standard neutral model (snm), which does not allow for changes in population size (Supplementary Note S6). We tested the fit to the data of the following two population (2D) models: split_mig, IM, and a special case of IM where migration rates are fixed at 0 . As in 
the case of the 1D models, all 2D models include a parameter for the rate of misidentification of the ancestral allele at each SNP. Split_ mig has four parameters: (i) nu1, the size of population 1 after the split, (ii) nu2, the size of population 2 after the split, (iii) $\mathbf{T}$, the time since the split, and (iv) $\mathbf{m}$, the symmetric migration rate between the populations subsequent to the split. IM has six parameters: (i) s, the proportion of the total population in population 1 after the split, with the proportion of the total population in population 2 being 1 -s, (ii) nu1, the final size of population 1 , (iii) nu2, the final size of population 2, (iv) $\mathbf{T}$, the time since the split, (v) $\mathbf{m 1 2}$, the migration rate from population 2 to population 1 , and (vi) $\mathbf{m} 21$, the migration rate from population 1 to population 2 .

Additional details on optimizing parameter estimates and quantifying their uncertainty are included as a supplement (Supplementary Note S6). Since the no-migration IM model is nested within the more general IM model, a likelihood ratio (LR) test can be used to determine if the model that allows migration is a significantly better fit (Supplementary Note S6).

The proportionate reduction in $N_{e}$ was estimated based on the relative frequency of the MAT-1 and MAT-2 alleles $\left(N_{e(m t)}\right)$, and the relative frequency of female-sterile strains and hermaphrodites $\left(N_{e(f)}\right)$ by using the equations of Leslie and Klein (1996).

Chromosomal distribution of nucleotide diversity and fixed and shared polymorphisms. Nucleotide diversity within and between species was calculated based on GBS haplotypes extracted from STACKS (Supplementary Note S7). The relative proportion of fixed differences and shared polymorphisms was computed across chromosomes (Supplementary Note S7). Fixed difference or shared polymorphism (FDSP) sites were ordered along the 12 chromosomes. Then, for each consecutive block containing 20 FDSP sites we computed the proportion that were fixed differences and the average chromosome position of these sites. From the largest 16 scaffolds, the 10 longest runs of fixed differences without shared polymorphisms and 10 longest runs of shared polymorphisms without fixed differences were identified.

Selective sweep scan. SweepFinder2 v1.0 (DeGiorgio et al. 2016) was used for a genome scan of each species to detect selective sweeps. These analyses were performed using all strains. We created input files starting with the 22,817 SNPs from the full set of strains from both species (Supplementary Note S7), and we
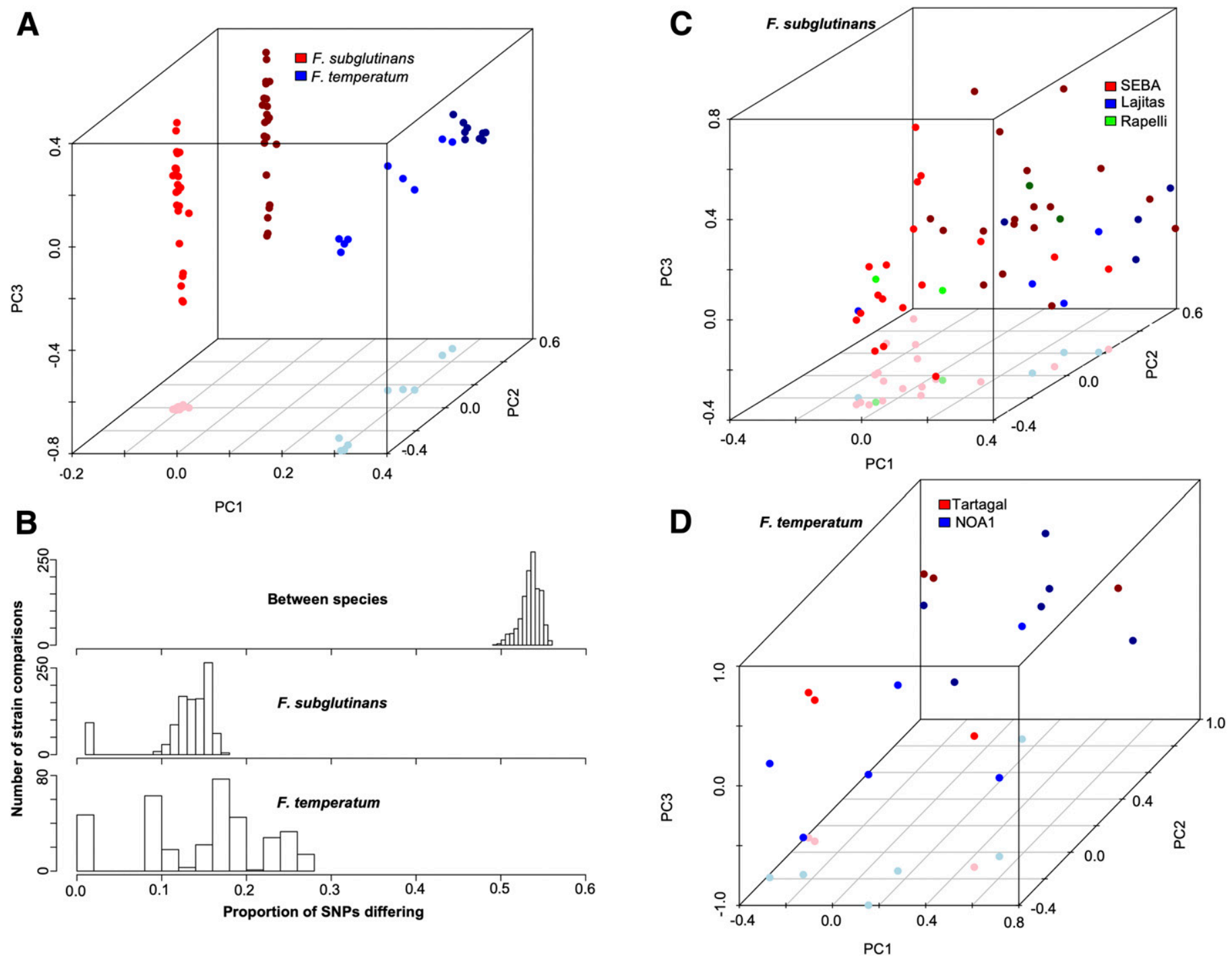

Fig. 1. Principal components and mismatch analyses of the sample. A, Principal component analysis (PCA) plot of the first three principal components for the set of 33 clone-corrected strains, with Fusarium subglutinans in red and F. temperatum in blue. To help visualize in three dimensions, the projections of the data points are shown on the PC1 $\times$ PC2 plane (lighter shades) and on the PC1 $\times$ PC 3 plane (darker shades), resulting in a total of $99(3 \times 33)$ points in the plot. B, Mismatch distribution of all pairwise strain comparisons, split into comparisons between-species, within F. subglutinans, and within F. temperatum. The $x$-axis corresponds to the proportion of all single nucleotide polymorphisms that differ between each strain pair. C, PCA plot of the first three principal components for the 24 clonecorrected strains of $F$. subglutinans. Projections of data points are included as in A. D, PCA plot of the first three principal components for the nine clone-corrected strains of F. temperatum. Projections of data points are included as in A. In C and D, different colors represent the geographic regions of origin for each strain. 
included information on the ancestral and derived states, when known (described under Demographic analysis above). SweepFinder 2 calculated an empirical allele frequency spectrum for the largest 15 scaffolds, and then conducted a scan for selective sweeps by comparison with the precomputed spectrum. We used the LR statistic computed for windows of $\sim 25 \mathrm{~kb}$ by SweepFinder 2 to rank the major peaks in the genome indicative of sweeps (Supplementary Note S7).

Data availability. The sequencing data, post-splitting by barcode and quality trimming, have been deposited in the NCBI Sequence Read Archive under BioProject ID PRJNA638806. The vcf file produced by GATK, various derivative SNP files used for specific analyses (e.g., PCA, STRUCTURE, and SweepFinder2), and custom Perl scripts written by the authors and cited in this manuscript are available at K-State's Research Exchange (K-REx) at https://krex.k-state.edu/dspace/handle/2097/40869 (Fumero et al. 2020b).

\section{RESULTS}

Strains of Fusarium were recovered from five regions in Argentina (Table 1, Supplementary Fig. S1). From a larger sample of strains, we retained 73 for analysis after filtering out strains with the following: (i) diagnostic $E F-1 \alpha$ gene sequences that were exact or close matches to sequences from species other than $F$. temperatum or $F$. subglutinans, (ii) different $E F-1 \alpha$ sequences when repeated from an independent DNA isolation, or (iii) GBS locus heterozygosity in excess of background thresholds (interpreted as contamination in these haploid species). Specifically, four strains with over 45 loci that appeared heterozygous (range 103 to 1671) and with over 11 of the above loci that appeared heterozygous uniquely in that strain (range 56 to 672) were excluded from the study due to concern that these samples had been contaminated by a second genotype at the culture or DNA library stage. Among the original strain set, the median number of loci that appeared heterozygous was 24 , while the median number of loci that appeared heterozygous only in a given strain was one.

The 73 strains included 11 previously identified to the species level (six F. subglutinans and five F. temperatum) (Fumero et al. 2015) that were used as reference strains to confirm the species assignment of the remaining field isolates. Based on partial $E F-1 \alpha$ gene sequences, 46 strains were identified as F. subglutinans and 27 as F. temperatum (Supplementary Table S1). Sexual crosses with tester strains confirmed species assignment in all cases, though two strains of $F$. subglutinans and one of $F$. temperatum additionally showed the ability to initiate production of perithecia when crossed with the tester strain from the other species (Supplementary Table $\mathrm{S} 1)$. All strains of $F$. subglutinans were from SEBA, Lajitas, or Rapelli, while all strains of $F$. temperatum were from Tartagal or NOA1, so the range of the two species did not overlap based on the strains included in this study.
Distribution of SNPs. To generate a set of SNPs with genotypes from strains in both species, we needed to map GBS reads from all samples to a single common reference genome. We chose the F. temperatum CMWF389 genome because it is the most highly assembled reference genome publicly available from either $F$. temperatum or $F$. subglutinans. The majority of strains of F. subglutinans mapped $>85 \%$ of their reads to this reference, and the majority of strains of $F$. temperatum mapped $>90 \%$ of their reads. The excess reads not mapping from strains of $F$. subglutinans, presumably from $F$. subglutinans-specific genome regions, are not expected to change the results reported here, which focus on the portions of the genomes common to and highly similar between both species.

GBS sequencing and mapping resulted in over 28,000 sequenced loci and identified SNPs throughout the genome. After dropping polymorphic INDELs and SNPs that were not biallelic, 22,817 biallelic SNPs were polymorphic in the 73-strain sample. These SNPs were found on 24 of the 25 longest scaffolds of the F. temperatum CMWF389 reference genome, including on all 22 scaffolds that are $>12 \mathrm{~kb}$. The 19 scaffolds with no GBS SNPs represent $<70 \mathrm{~kb}$, or $<0.2 \%$ of the total genome. The median size of intervals between adjacent SNPs was $46 \mathrm{bp}$, with $31 \%$ of intervals $>1 \mathrm{~kb}$ but only $5 \%$ of intervals $>10 \mathrm{~kb}$. The number of SNPs on each scaffold in the reference genome was roughly proportional to the scaffold size, with a SNP density of 5 per $10 \mathrm{~kb}$ of reference sequence. Scaffolds 8, 10, 12, 14, and 15 had the highest SNP density, at $0.67,0.78,0.67,0.8$, and $0.74 \mathrm{SNPs} / \mathrm{kb}$ respectively, and Scaffolds 1, 2, 3, and 11 had the lowest at $0.39,0.44,0.45$, and 0.42 $\mathrm{SNPs} / \mathrm{kb}$, respectively. The average SNP density calculated across only the sequenced GBS loci is much higher ( $\sim 9 \mathrm{SNPs} / \mathrm{kb})$, as our GBS loci cover $\sim 2.5 \mathrm{Mb}$, or $1 / 18$ of the whole reference genome.

The SNP data are characterized by fixed differences (monomorphic sites within species but with different alleles in the two species), shared polymorphisms (sites with the same two alleles segregating in both species), and sites polymorphic in one of the species and fixed in the other. Of the 17,619 SNPs with a genotype call from at least five strains of $F$. temperatum and nine strains of F. subglutinans, 3,752 were fixed differences between the two species, 2,521 were polymorphic in both species, 5,844 were polymorphic only in $F$. subglutinans, and 5,502 were polymorphic only in F. temperatum.

Clusters of closely related strains or clonal lineages were detected within each species. For $F$. subglutinans, eight clonal lineages were identified: five pairs of strains, one cluster of three strains, one cluster of four strains, and one cluster of 13 strains (Supplementary Table S1). Clonal lineages usually originated from the same region, but not always. For one pair of closely related strains, one strain was from SEBA and the other from Lajitas (cluster III, Supplementary Table S1), more than $1,500 \mathrm{~km}$ away. This case could result from a migration event, i.e., movement of an asexual clone between these regions or from a third location possibly through movement of seed.

TABLE 1. Geographical and climatic characteristics of the sampling regions

\begin{tabular}{|c|c|c|c|c|c|c|c|}
\hline Region & F. subglutinans & F. temperatum & Latitude longitude $^{a}$ & Elevation (masl) ${ }^{\mathrm{a}}$ & $\begin{array}{c}\text { Mean annual } \\
\text { temperature }\left({ }^{\circ} \mathrm{C}\right)^{\mathrm{b}}\end{array}$ & $\begin{array}{c}\text { Mean annual } \\
\text { precipitation }(\mathrm{mm})^{\mathrm{b}}\end{array}$ & Type of maize ${ }^{c}$ \\
\hline SEBA $^{\mathrm{d}}$ & 25 & - & $37^{\circ} 49^{\prime} 00^{\prime \prime} \mathrm{S} 58^{\circ} 15^{\prime} 00^{\prime \prime} \mathrm{W}$ & 97 & 13.9 & $650-900$ & Commercial hybrid \\
\hline Lajitas & 18 & - & $24^{\circ} 41^{\prime} 00^{\prime \prime} \mathrm{S} 64^{\circ} 15^{\prime} 00^{\prime \prime} \mathrm{W}$ & 455 & 21.1 & $650-800$ & Commercial hybrid \\
\hline Rapelli & 3 & - & $26^{\circ} 23^{\prime} 31^{\prime \prime} \mathrm{S} 64^{\circ} 30^{\prime} 14^{\prime \prime} \mathrm{W}$ & 403 & 20.3 & $700-800$ & Commercial hybrid \\
\hline Tartagal & - & 9 & $22^{\circ} 30^{\prime} 00^{\prime \prime} \mathrm{S} 63^{\circ} 50^{\prime} 00^{\prime \prime} \mathrm{W}$ & 490 & 20.4 & 500 & 'Perla' \\
\hline NOA $1{ }^{\mathrm{e}}$ & - & 18 & $23^{\circ} 11^{\prime} 59.5^{\prime \prime} \mathrm{S} 65^{\circ} 20^{\prime} 55.9^{\prime \prime} \mathrm{W}$ & 3,012 & 11.7 & 400 & Andean \\
\hline
\end{tabular}

a Online geographic tool GeoHack from Wikipedia: https://geohack.toolforge.org/.

b Online climatic tools https://es.weatherspark.com and https://es.climate-data.org.

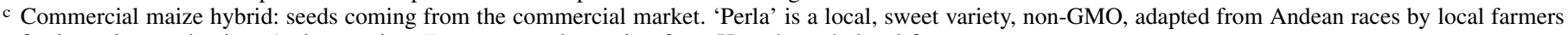
for intensive production. Andean maize: Zea mays seeds coming from Humahuaca's local farmers.

d SEBA: region including three locations in the southeast portion of Buenos Aires province (Balcarce, Necochea, and Lobería).

e NOA1: region including three locations in the Jujuy province (Uquía, Bárcena, and Humahuaca). 
For F. temperatum, six clonal lineages were identified: two pairs of strains, and clusters of three, four, six, and seven strains. Regarding migration, the cluster of three contained two strains from Tartagal and one from NOA1, which is $170 \mathrm{~km}$ away, and a second cluster contained six strains from Tartagal and one from NOA1 (Supplementary Table S1). These migrations could not have occurred via seed as the varieties planted in these regions are different. Additionally, the clusters of four and six strains each had members from all three of the geographically close NOA1 sampling sites. When all but a single strain are dropped from each clonal lineage, a clone-corrected sample of nine strains of $F$. temperatum and 24 strains of $F$. subglutinans, or 33 total strains, remains.

Evidence for recombination. Sexual crosses with these species can be made readily in the lab, but the finding that a sizeable proportion of field strains belong to clonal lineages suggests the data's fit to models incorporating sexual recombination and models of pure clonal reproduction must be assessed. The negative correlation between the LD measure $r^{2}$ and the distance between SNPs supports the hypothesis that sexual outcrossing occurs in both species (Miyashita and Langley 1988; Schaeffer and Miller 1993). For $F$. subglutinans, the Pearson's correlation coefficient is -0.28 and the Spearman's rank correlation coefficient between $r^{2}$ and the distance between SNPs is -0.24 , and $r^{2}$ decays to its midpoint within 20 to $30 \mathrm{~kb}$ (Fig. 2). In F. temperatum, the Pearson's and Spearman's rank correlation coefficients are both -0.21 , with the $r^{2}$ midpoint at $\geq 60 \mathrm{~kb}$. Following the procedure of Piganeau and Eyre-Walker (2004), we assessed the significance of the relationship between LD and distance in each species by performing a Mantel test. For both species and both correlation coefficients, none of 1,000 permuted datasets shows a correlation approaching the observed values (none are less than -0.0102 , compared with values less than -0.2 in the observed data), so for both species and both correlation coefficients, $P<0.001$ and the hypothesis of the absence of recombination can be rejected.

Background levels of LD for unlinked SNPs are substantially higher in F. temperatum (Fig. 2). Two factors likely contribute to this observation. First, the small size of the clone corrected

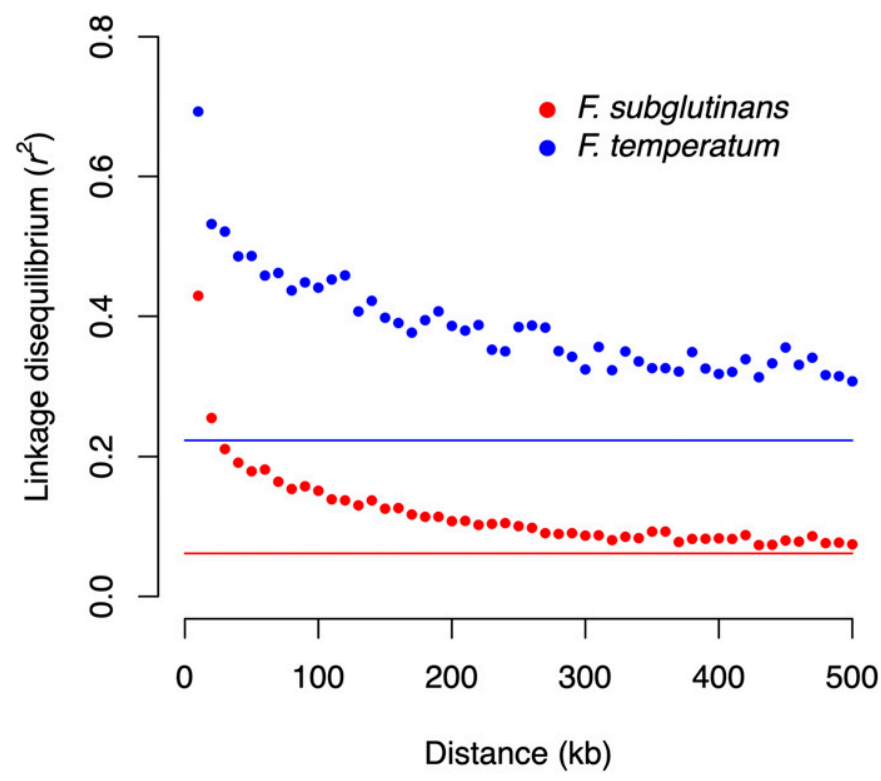

Fig. 2. Linkage disequilibrium decay with inter-single nucleotide polymorphism (SNP) distance for each species. The mean of linkage disequilibrium measure $r^{2}$ for all pairs of SNPs with distance between falling within a particular $10 \mathrm{~kb}$ window. Only genotypes from the 24 and 9 strains retained after clone correction of Fusarium subglutinans and F. temperatum, respectively, were used in the calculation of $r^{2}$. The horizontal lines, colored to correspond to each species, give the mean $r^{2}$ for all pairs of SNPs found on different scaffolds and therefore presumably unlinked.
F. temperatum sample $(n=9)$ increases the average $r^{2}$ for unlinked SNPs, as the small sample size limits the number of ways that alleles can be randomly placed into two-locus haplotypes. Second, if the population has genetic substructure, then residual LD is expected even for unlinked SNPs.

To provide further support for recombination in the field in both species, we estimated the minimum number of recombination events in the history of the clone-corrected samples from each species using the software RecMin (Myers and Griffiths 2003). Over 600 recombination events were estimated in each species (Supplementary Table S3). The minimum number of recombination positions $\left(R_{m}\right)$ and recombination events $\left(R_{h}\right)$ were approximately two and three times higher, respectively, in $F$. subglutinans than in F. temperatum. The small size of the clone-corrected sample (nine strains) in F. temperatum more severely limits the amount of information available about the recombination history (Myers and Griffiths 2003). The number of recombination events inferred per reference genome scaffold is roughly proportional to size, and the number of additional events detected by $\mathrm{R}_{\mathrm{h}}$ over $\mathrm{R}_{\mathrm{m}}$ is greater in $F$. subglutinans, as expected due to its larger sample size.

Species boundary between $F$. subglutinans and $F$. temperatum. We investigated the species boundary between the two species by computing mismatch distributions and performing PCA and multiple clustering analyses.

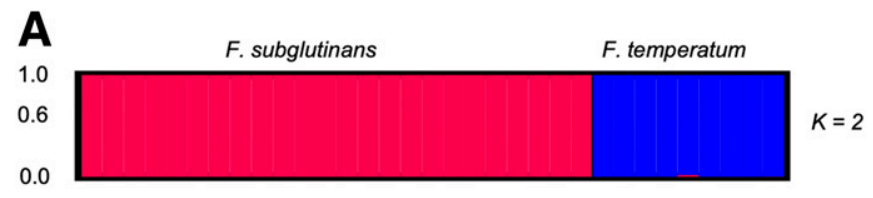

B

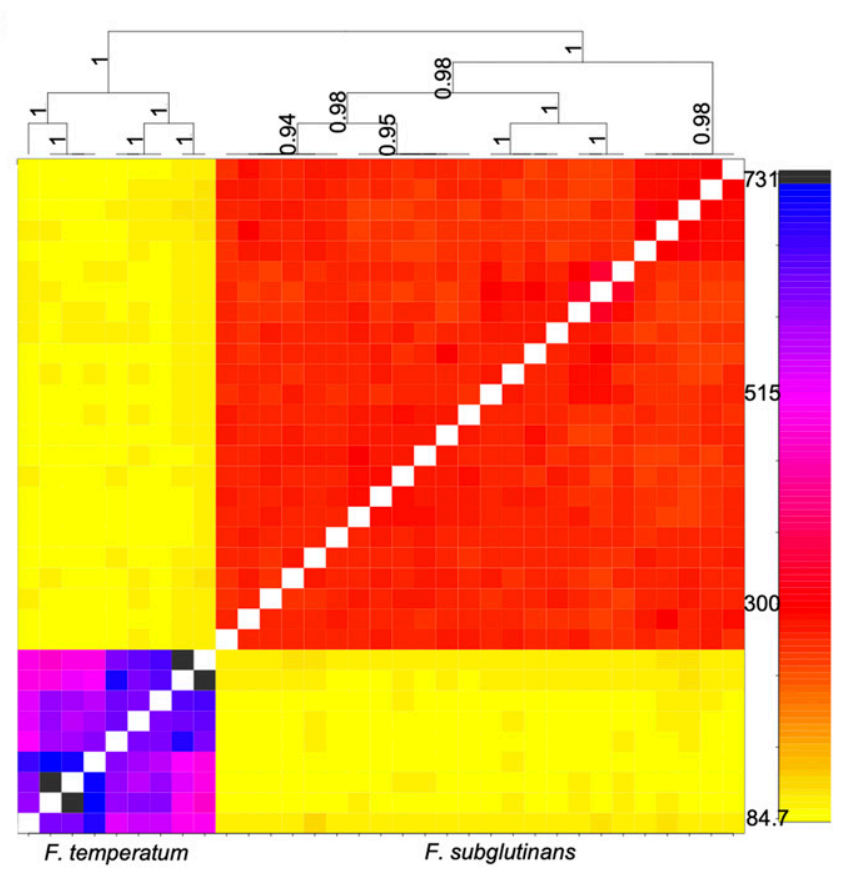

Fig. 3. Population structure of the sample. A, Summary of STRUCTURE runs assuming $K=2$ clusters for the clone-corrected set of 33 strains support the separation between both species. Each strain is represented by a column along the $x$-axis and is partitioned into K colored segments according to each strain's estimated membership fraction in each of the $\mathrm{K}$ inferred genetic clusters. B, Clustered fineRADstructure coancestry matrix heatmap, indicating pairwise coancestry between strains of Fusarium subglutinans and F. temperatum after clone correction. Inferred tree relating inferred populations is shown at the top with posterior assignment probabilities. For each row, cell color indicates probability of coancestry of each strain with the white cell. Yellow indicates lowest probability of coancestry, and red and blue indicate higher probabilities. The figure shows a clear split between $F$. temperatum and $F$. subglutinans, as well as an indication of within species substructure. 
The separation between $F$. subglutinans and F. temperatum was supported by PCA using the clone-corrected sample (Fig. 1A). The first principal component, $\mathrm{PC} 1$, resolved all of the strains into one of two species clusters with no overlaps and explains $43 \%$ of the total variance (Tracy-Widom test $P=0.001$; analysis of variance test of population differences along PC1 $P<10^{-6}$ ). Principal components 2 (PC2) and 3 (PC3) explain an additional 6.7 and $3.5 \%$ of the total variance, respectively. PC2 groups strains of $F$. subglutinans into a single tight cluster, but disperses strains of $F$. temperatum, while PC3 groups strains of $F$. temperatum and disperses strains of F. subglutinans.

The mismatch distribution similarly clearly resolved the two species. The distribution when comparing any one strain of $F$. temperatum with any one strain of $F$. subglutinans was unimodal, with a mode of approximately $54 \%$ of the SNPs that differed, and a range between 49.5 and $56 \%$ (Fig. 1B). In contrast, no more than $28 \%$ of SNPs differed between strains belonging to the same species (Fig. 1B).

The software STRUCTURE was used to infer population structure based on both the 12,021 SNPs from the clone-corrected set of 33 strains and the 22,817 SNPs from the full set of 73 strains. In each case, models with the number of subpopulations $K=1$ through 20 were analyzed. The Evanno method (Evanno et al. 2005) was used to infer the $K$ with greatest posterior probability. For both the clonecorrected and full sample sets, $K=2$ was the best fit for the data (Supplementary Table S4). At $K=2$, all strains of $F$. subglutinans had $>99.5 \%$ membership in the same subgroup, Q2, in both the clonecorrected and the full sample set (Fig. 3A, Supplementary Fig. S2a, Supplementary Table S1). For $K=2$ in the clone-corrected set, eight strains of $F$. temperatum had $>99.5 \%$ membership in Q1, and the ninth strain had $>98 \%$ membership in Q1 (Fig. 3A, Supplementary Table S1). For the full set of strains of F. temperatum, 20 had $>99.5 \%$ membership in Q1, while the remaining seven had at least $86 \%$ membership in Q1 (i.e., maximum 14\% membership in Q2; Supplementary Fig. S2a, Supplementary Table S1).

We also inferred population structure based on nearest neighbor coancestry of GBS haplotypes using the software fineRADstructure. The coancestry matrix and tree inferred for both the complete and the clone-corrected strain sets clearly resolved $F$. subglutinans and F. temperatum, with uniformly low coancestry inferred across species compared with within species (Fig. 3B, Supplementary Fig. S3).

Population structure within species. Despite the relatively small sample sizes and the presence of several clonal lineages, multiple analyses are consistent with greater population substructure within $F$. temperatum than within $F$. subglutinans.

The first three PCs explained 10, 8, and $5 \%$ of the variance from the clone-corrected set of 24 strains of $F$. subglutinans. Wellseparated clusters are difficult to define, though PC1 separates three strains from Lajitas and three from SEBA with high values from the other strains (Fig. 1C). The diffuse group that contains the remaining 18 strains included the majority of strains from SEBA, all strains from Rapelli, one strain from Lajitas and the strain representing the SEBA-Lajitas clonal lineage (lineage III in Supplementary Table S1). The PCA plot for the full set of 46 strains of $F$. subglutinans was strongly affected by the presence of clonal lineages, with most strains in a single large group with three outlier clusters that each correspond to a different clonal lineage (Supplementary Fig. S2c).

In a similar analysis of the F. temperatum data, the first three PCs explained 35,13 , and $13 \%$ of the total variance from the clonecorrected set of nine strains. PC1 spread out the strains into three groups: a group of four strains from both locations; a group of three from NOA1; and a pair of strains, one each from NOA1 and Tartagal (Fig. 1D). PC2 separated one NOA1 strain from the group of three mentioned previously from the other eight strains. The PCA plot for the full set of 27 strains of $F$. temperatum also was affected by the presence of clonal lineages, though the outlier clusters cannot be explained by single clonal lineages (Supplementary Fig. S2d).
Analysis of SNP mismatch distributions within each species is consistent with greater population substructure in $F$. temperatum. For the 27 strains of $F$. temperatum, the mismatch distribution is multimodal, even when comparisons of strains within the same clonal lineage are disregarded. The distribution spans from 8.7 to $27 \%$ of SNPs differing and has modes at 9, 17, and 25\% (Fig. 1B). For $F$. subglutinans, the mismatch distribution is unimodal once the clonal lineages are accounted for, with a mode at approximately $15 \%$ of SNPs differing and a range from 9 to $17.5 \%$ (Fig. 1B).

Based on the mismatch distributions, genetic subpopulations within species do not correspond well to geographic location. For each species, within location comparisons were, on average, only slightly more similar than between location comparisons. The ranges of the proportion difference across the within-location and between-location comparisons were largely overlapping.

Analysis of nearest neighbor coancestry within species with fineRADstructure also supports the conclusion of more population substructure in F. temperatum than in F. subglutinans. After clone correction in $F$. subglutinans, each strain has a similar level of coancestry with all other strains, and no subgroup had obviously stronger blocks of shared coancestry (Fig. 3B). Without clone correction, traces of higher coancestry are mainly limited to clonal lineages (Supplementary Fig. S3). After clone correction in F. temperatum, two groups of strains had much stronger shared within-group than between-group coancestry. These groups did not correspond to geographic regions (Fig. 3B). This clustering also was detected when the full set of strains was analyzed (Supplementary Fig. S3).

Demographic inference with $\delta \mathbf{a} \mathbf{i}$. Demographic modeling of the joint allele frequency spectrum was used to test two hypotheses for the high number of shared polymorphisms: (i) a recent separation of the species, and (ii) the occurrence of gene flow between the species after the split. Our previous analyses clearly separated the two species based on a large number of fixed differences. No obvious hybrids were seen in our strain sample, but hybridization and potential gene flow between the species during their divergence remains a possibility.

Demographic parameters related to the two species and their divergence were inferred using the diffusion-based approach implemented in the software package $\delta a \delta i$ (Gutenkunst et al. 2009). The number of ancestral-state inferred SNPs with no missing genotype data were maximized by projecting samples sizes down to nine strains of $F$. subglutinans and five strains of $F$. temperatum, leaving 6,871 SNPs for demographic analysis. These 6,871 SNPs represent an effective length queried of approximately $769 \mathrm{~kb}$ per strain. The $F_{S T}$ estimated using the method of Weir and Cockerham (1984) as implemented in $\delta a \delta i$ for the combined sample of both species $(n=14)$ was 0.53 .

Based on marginal analyses of the allele frequency spectra in each species, present day effective population sizes $\left(N_{e}\right)$ of each species are smaller than they were in the past. Models of exponential population size change or instantaneous change (two epoch and three epoch models in $\delta a \delta i$ ) indicate a smaller $N_{e}$ for current populations and fit the data better than the standard neutral model, which does not allow for population size changes. The small postprojection sample sizes prevented an evaluation of potential population splits within each species. The fit to the observed joint allele frequency spectrum of both species was tested for three models (Table 2): (i) split_mig, in which an ancestral population is split into two populations of specified size, with migration subsequent to the split, (ii) an isolation-with-migration (IM) model with exponential population growth subsequent to the split, and (iii) a special case of the IM model where no migration occurs between the species. Models (i) and (ii) have two main differences. split_mig models symmetric migration between species while IM allows asymmetric migration. Also, split_mig models the population size change that is independent for each species as instantaneous at the time of the split, while IM models the split such that immediately 
following it the sum of the sizes of the resulting populations equals the ancestral population size, but subsequently each resulting population can experience exponential growth independently. Models (i), (ii), and (iii) had log likelihoods of $-274,-257$, and -336 , respectively, making IM the best fitting model.

Focusing on the IM model, the optimized population mutation rate parameter $\theta=4 N_{e} \mu$ (where $\mu$ is the per generation mutation rate) for the ancestral population was 3,441 or 0.0045 per bp. If it is assumed that the per base pair per generation mutation rate is $7.82 \times 10^{-9}$ (previously estimated for Neurospora, Ellison et al. 2011) and one generation equals one year, then the $\theta$ estimate corresponds to a value of $>143,000$ for $N_{e}$ of the ancestral population. Additional optimized IM parameters include $s$ (proportion of $F$. subglutinans in the total two-species population after the split) $=0.56$ and time since split $T=0.56$ in units of $2 \times N_{e}$ of the ancestral population generations, which suggest the ancestral population split into two populations (i.e., species) of roughly equally size $\sim 160,000(0.56 \times 2 \times \sim 143,000)$ years ago. Even though uncertainty exists about the divergence time, especially due to uncertainty in the corresponding mutation rate, the time estimate is not particularly recent and substantially precedes maize domestication (Matsuoka et al. 2002).

Other optimized IM parameters include: the final $F$. subglutinans $N_{e}$ relative to ancestral $N_{e}, n u_{1}=0.11$; the final F. temperatum $N_{e}$ relative to ancestral $N_{e}, n u_{2}=0.10 ; 2 \times N_{e}$ of the ancestral population $\times$ the per individual per generation migration rate into $F$. subglutinans, or $m_{12},=0.28$; and the analogous migration rate into $F$. temperatum, $m_{21}=0.67$. These values are similar to the optimized parameters from the split_mig model, where the symmetric migration rate, 0.39 , was intermediate between the two asymmetric migration rates from IM. These parameters suggest that the $N_{e}$ of the current species are both about $10 \%$ of the ancestral $N_{e}$, consistent with the previous single population analyses. Notably, the migration (gene flow) rate from $F$. subglutinans into $F$. temperatum has been over twice the reverse rate, with this biased gene flow strongly supported by the $95 \%$ confidence intervals (Table 2). Based on a likelihood ratio test (adjusted LR test score 19.2, $P<10^{-4}$ ), the full IM model is a significantly better fit than the reduced parameter IM model that lacks migration (Fig. 4A and $\mathrm{B}$ ). Thus, the model provides strong evidence for interspecific gene flow subsequent to the estimated divergence time. The IM model generally provides a good fit to the data, as seen in the residuals plot (Fig. 4A), with the poorest fit owing to the data's excess of derived alleles that are found in both species at low frequency.

Effects of MAT allele ratio and female fertility on $\boldsymbol{N}_{e}$. The mating-type ratio for $F$. subglutinans was not significantly different from $1: 1\left(\chi^{2}=0.78, P>0.05\right)$ and is only expected to reduce $N_{e}$ to $98.3 \%$ of the value expected had the ratio been equal (Table 3 ). However, of the 46 strains, only three were female-fertile, resulting in a reduction of $N_{e}$ to $23 \%$ of the value expected had all strains been female-fertile (Table 3). Had all strains been female-fertile, this would translate to a higher actual $N_{e}$ for the population and resulted in more genetic variation. Given that our $\delta \mathrm{a} \delta$ i estimate of $N_{e}$ for F. subglutinans is $\sim 15,600(0.109$ relative size from Table $2 \times$ ancestral population of 143,000$)$, then had all strains been femalefertile we would expect more genetic variation and a proportionally larger estimate from $\delta a \delta i$ of $N_{e} \sim 68,000(15,600 / 0.23)$.

The mating-type ratio for $F$. temperatum was 4:23 (MAT-1:MAT$2)$, which is significantly different from $1: 1\left(\chi^{2}=6.69, P<0.05\right)$, and is expected to reduce $N_{e}$ to $50 \%$ of the value expected had the ratio been equal. Of the 27 strains, only five were female-fertile, resulting in a reduction of $N_{e}$ to $53 \%$ of the value expected had all strains been female-fertile (Table 3). Using similar reasoning as for $F$. subglutinans, had all strains been female-fertile we would expect more genetic variation and a proportionally larger estimate from $\delta$ adi of $N_{e} \sim 28,000(0.103 \times 143,000 / 0.53)$. And, had all strains been female-fertile and the MAT alleles been present at an equal frequency as well, and assuming that the $N_{e}$ reductions due to female sterility and mating type skew act independently, we would expect an estimate from $\delta$ a $\delta$ i of current $N_{e}>55,000(\sim 28,000 / 0.5)$.

For both species, the proportional reduction in $N_{e}$ due to lack of female-fertile strains can be used to estimate the number of asexual generations per sexual generation (Table 4) after making assumptions about the selective disadvantage, $\Theta$, of hermaphroditism during asexual propagation and the mutation rate, $\mu$, to female sterility (Leslie and Klein 1996). The estimated number of generations is somewhat higher for $F$. subglutinans due to the lower female fertility observed, though given the small sample sizes and low fertility common to both species, the fertility rates of the two species are not significantly different from one another $\left(\chi^{2} P>\right.$ $0.05)$. The inverse of the number of generations for a cycle gives the average proportion of the population going through sexual reproduction at any one time, and the average portion of the population reproducing sexually per generation based on this set of strains is $\sim 1 \%$ when $(1-\mu) \Theta$ is 0.98 or 0.99 .

The estimated frequency of female fertility in these populations is low relative to previous estimates from the FFSC (Leslie and Klein 1996), with estimates from $F$. verticillioides and $F$. proliferatum greater, and the estimate from $F$. thapsinum of $\sim 10 \%$ similar to those of $F$. temperatum and $F$. subglutinans. Given the small sample sizes for the populations in the current study and the similarly low

TABLE 2. $\delta \mathrm{a} \delta \mathrm{i}$ parameters for $2 \mathrm{D}$ models

\begin{tabular}{|c|c|c|c|c|c|c|c|c|c|}
\hline Parameters & $\begin{array}{c}\text { Log } \\
\text { likelihood } \\
\text { optimized } \\
\text { model }\end{array}$ & Theta $^{\mathrm{a}}$ & $\begin{array}{c}\text { Ratio of } \\
\text { Fusarium } \\
\text { subglutinans to } \\
\text { combined sizes } \\
\text { of both species } \\
\text { after split }\end{array}$ & $\begin{array}{l}\text { F. subglutinans } \\
\text { final size }\end{array}$ & $\begin{array}{l}\text { F. temperatum } \\
\text { final size }\end{array}$ & $\begin{array}{l}\text { Time } \\
\text { since } \\
\text { split }^{\mathrm{c}}\end{array}$ & $\begin{array}{l}\mathrm{m} 12 \text { (migration into } \\
\text { F. subglutinans) }\end{array}$ & $\begin{array}{l}\mathrm{m} 21 \text { (migration into }^{\mathrm{d}} \\
\text { F. temperatum) }\end{array}$ & $\begin{array}{c}\text { Mis-inferred } \\
\text { ancestral } \\
\text { state }^{\mathrm{e}}\end{array}$ \\
\hline Split_mig & -274.425 & $3,874.70$ & $\mathrm{na}^{\mathrm{f}}$ & 0.106 & 0.119 & 0.237 & 0.393 & 0.393 & $4.91 \times 10^{-6}$ \\
\hline $\mathrm{IM}(\mathrm{mig}=0)$ & -336.493 & $3,757.80$ & 0.104 & 0.091 & 0.034 & 0.161 & 0.000 & 0.000 & $1.43 \times 10^{-45}$ \\
\hline IM & -257.210 & $3,440.85$ & 0.560 & 0.109 & 0.103 & 0.555 & 0.280 & 0.668 & $2.06 \times 10^{-4}$ \\
\hline LCI IM ${ }^{\mathrm{i}}$ & & $3,067.76$ & 0.402 & 0.088 & 0.080 & 0.400 & 0.190 & 0.475 & 0.000 \\
\hline UCI IM ${ }^{\mathrm{i}}$ & & $3,813.94$ & 0.717 & 0.130 & 0.125 & 0.711 & 0.370 & 0.861 & 0.025 \\
\hline
\end{tabular}

a Per length of genome analyzed.

b Relative to ancestral $N_{e}$.

c In units of $2 \times$ ancestral $N_{e}$ generations.

d $2 \times$ ancestral $N_{e} \times$ per individual per generation migration rate.

e Per polymorphic site rate of mis-inference.

${ }^{f}$ Not applicable.

g Standard deviation for IM parameters generated from the Godambe information matrix (GIM).

${ }^{\text {h }}$ Coefficient of variation for IM parameters.

${ }^{i}$ Upper and lower $95 \%$ confidence intervals for each parameter. 
fertility rates $(<20 \%$ in each case), the female fertility data are not sufficient to conclude that the frequency of sexual reproduction in F. temperatum is different from that in F. subglutinans in Argentina. The low female fertility, the number and size of clonal lineages, and their geographic distribution are consistent with asexual reproduction being much more common than sexual reproduction in both these fungi in Argentina. This conclusion is not inconsistent with the evidence of abundant recombination within each species, which demonstrates that even reduced levels of sexual reproduction and recombination can have an important impact on the pattern of population variation.

Genome-wide distribution of genetic diversity, fixed differences, and shared polymorphisms. The ratio of fixed differences to shared polymorphisms was computed across the genome to scan for regions with excesses of either fixed differences or shared polymorphisms. Regions with an excess of fixed differences (islands of divergence) may be important in maintaining barriers between species. Regions with an excess of shared polymorphisms can
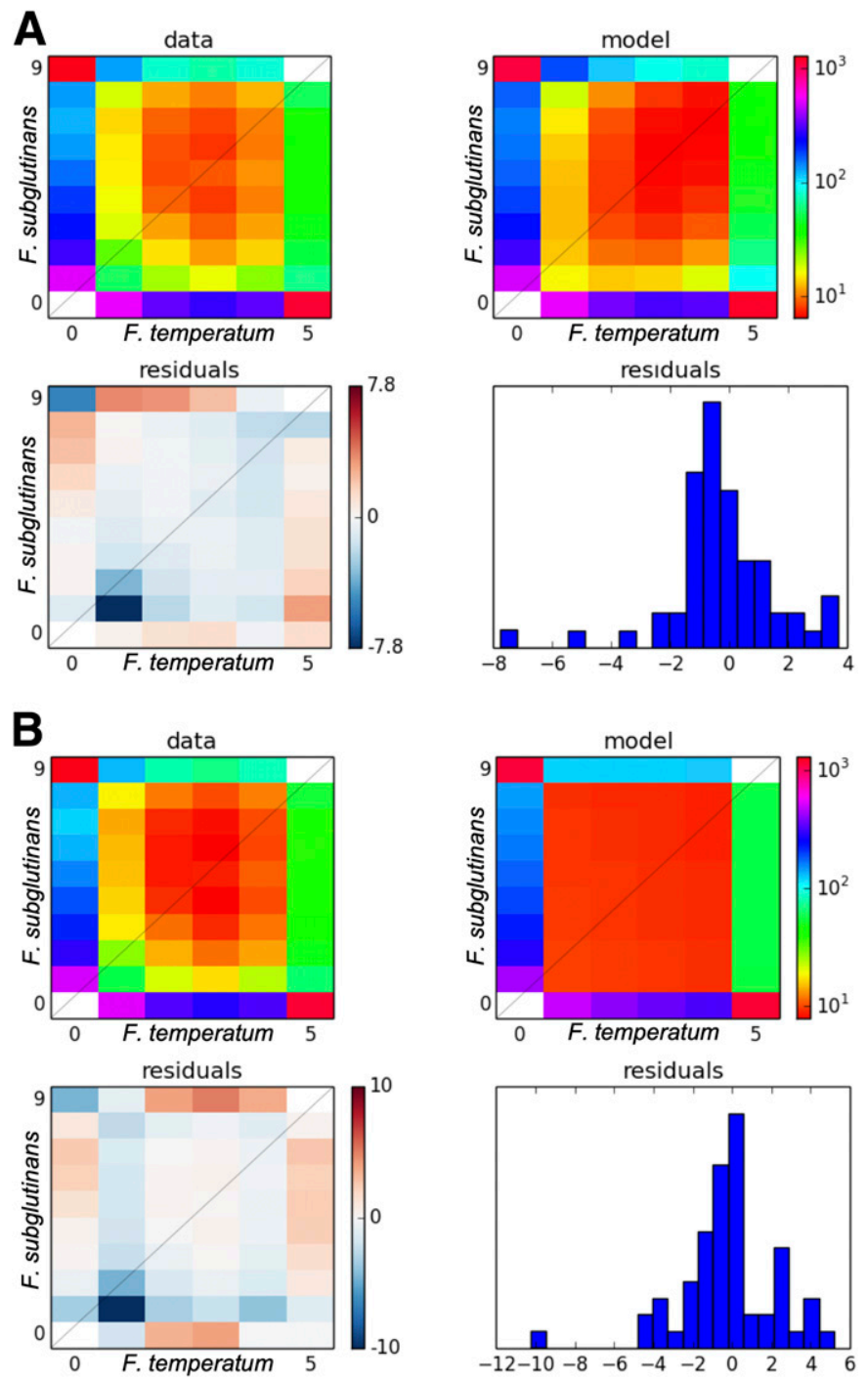

Fig. 4. Comparison of the joint allele frequency spectrum with that expected under isolation with migration (IM) demographic models. A, Full IM model. $\mathbf{B}$, IM model where migration is prevented. Upper panels of $\mathbf{A}$ and $\mathbf{B}, x$-axis shows derived allele count for Fusarium temperatum, and $y$-axis shows derived allele count for F. subglutinans. Colors represent the scaled count of single nucleotide polymorphisms with each possible two population allele count. The observed joint allele frequency spectrum is displayed in the left panel and the expected frequency spectra from tested models are on the right. Bottom plots in $\mathbf{A}$ and $\mathbf{B}$ show a residuals heatmap for each cell (left), and the same residuals are shown as a histogram (right). result from long-term balancing selection or when newly arisen alleles are advantageous in both species and have a gene flow advantage.

Both fixed differences and shared polymorphisms are interspersed throughout most of the genome, clearly indicating recombination, with $1.5 \times$ as many fixed differences as shared polymorphisms (Fig. 5). This relatively high proportion of fixed differences supports the hypothesis that $F$. subglutinans and F. temperatum are distinct species. Discrete, obvious genomic islands of differentiation or introgression were not apparent, which is not surprising given the relatively old divergence inferred from the demographic modeling since divergence islands become more difficult to detect over time as divergence increases genome-wide. The distribution of shared polymorphisms and fixed differences, however, follows a regular pattern as distal regions of chromosomes are enriched for shared polymorphisms and more proximal chromosome regions are enriched for fixed differences. The shortest chromosome, 12, is an exception with a low density of fixed differences across its length. The 10 longest intervals between shared polymorphisms generally occur in the middle of chromosomes, with only two coming within $1 \mathrm{Mb}$ of a chromosome end (on chromosomes 2 and 5). Conversely, the 10 longest intervals between fixed differences are found exclusively at the distal regions of chromosomes.

Local nucleotide diversity $(\pi)$, both within and between species, was calculated in windows across the genome. As previously observed in other Fusarium species (Chiara et al. 2015; Cuomo et al. 2007; Walkowiak et al. 2016), both measures tend to be highest at chromosome ends (Supplementary Fig. S4). On average, genomewide interspecies divergence is four times higher than intraspecies $\pi$. Intraspecies $\pi$ never exceeds interspecific divergence for the $100 \mathrm{~kb}$ genome windows with sufficient GBS sequence data for analysis (those that contain at least 250 bp of GBS loci). However, intraspecies $\pi$ is $>80 \%$ of interspecies divergence in several regions. Interspecific divergence is unusually low at the beginning of chromosome 3 and chromosome 11, the end of chromosome 5, and on chromosome 4 at $3.7 \mathrm{Mb}$ and chromosome 8 at $0.5 \mathrm{Mb}$. The regions on chromosomes 3,4 , and 8 correspond roughly to long runs of shared polymorphisms (Fig. 5). In addition, relative to divergence, $\pi$ is unusually high within $F$. subglutinans at the end of chromosome 8 , and within F. temperatum on chromosome 10 at 2.1 $\mathrm{Mb}$ and on chromosome 12 at 0.6 and $1.0 \mathrm{Mb}$.

Genome scans for detection of selective sweeps. To detect hard selective sweeps, we scanned our data from each species using SweepFinder2 (DeGiorgio et al. 2016) to detect genomic regions where the SNP allele frequency spectrum locally deviates from the genome-wide spectrum. The LR statistic probability of a sweep was plotted for windows of $\sim 25 \mathrm{~kb}$ along each chromosome (Fig. 5). One region has moderate evidence of a sweep common to both species, on chromosome 6 at $\sim 1.1 \mathrm{Mb}$. Seven additional peaks corresponding to putative selective sweeps in F. temperatum are found on chromosomes 1, 2, 3, 4, 6, and 11 (two peaks). F. subglutinans has only one additional peak on chromosome 9.

TABLE 3. Impact of unequal mating-type ratio and lack of female fertility on $N_{e}$ for Fusarium subglutinans and $F$. temperatum populations from maize in Argentina

\begin{tabular}{lccccc}
\hline Species & $N$ & MAT-1:MAT-2 & $N e_{(m t)}{ }^{\mathrm{a}}$ & $N_{f s}: N_{h}{ }^{\mathrm{b}}$ & $N e_{(f)}{ }^{\mathrm{c}}$ \\
\hline F. subglutinans & 46 & $26: 20$ & $45.2(98 \%)$ & $43: 3$ & $10.6(23 \%)$ \\
F. temperatum & 27 & $4: 23$ & $13.6(50 \%)$ & $22: 5$ & $14.2(53 \%)$ \\
\hline
\end{tabular}

a $N e_{(m t)}$ : effective population number based on mating type and expressed as a proportion of the maximum value (Leslie and Klein 1996).

b $N_{f s}$ : number of female-sterile strains in the sample. $N_{h}$ : number of femalefertile strains (hermaphrodites) in the sample.

c $N e_{(f)}$ : effective population number based on the number of strains that were hermaphrodites and expressed as a proportion of the maximum value (Leslie and Klein 1996). 


\section{DISCUSSION}

This study sheds important new light on the species boundary between $F$. subglutinans and $F$. temperatum. Distinguishing $F$. subglutinans and F. temperatum with morphological characters is difficult to impossible (Fumero et al. 2015; Scauflaire et al. 2011). Separating them based on the biological species concept is not always clear either, due to intraspecific variation in female fertility levels commonly seen in populations of many species in the FFSC (Leslie and Klein 1996), and the limited cross-species fertility observed for at least some members of these species (Fumero et al. 2015; Scauflaire et al. 2011; Steenkamp et al. 2002). The known interspecific crosses are at the least fertile end of the fertility spectrum for fungal crosses described by Perkins (1994).

Distinguishing these entities as phylogenetic species by using DNA sequence analysis usually is clear, if the number of loci involved is large enough. Prior to the recognition of these groups as two distinct species, they were described as cryptic species in the process of diverging based on the sequence of six loci from 29 strains (Steenkamp et al. 2002). Yet this same study found a total of seven phylogenetically distinct lineages across the two cryptic species, with no evidence of genetic recombination between lineages, leading to the conclusion that sexual reproduction was absent or extremely rare within the cryptic species and the suggestion that each lineage was in the process of separating into a discrete taxon (Steenkamp et al. 2002). Analysis of the two species with AFLPs found that their genetic similarity estimated with the Dice coefficient was $\sim 50 \%$ (Scauflaire et al. 2011), a level of similarity higher than most other comparisons between species in the FFSC, which rarely exceed $40 \%$, but less than the $70 \%$ similarity that usually identifies strains in the same species (Marasas et al. 2001).

In the present study, we used thousands of SNPs efficiently genotyped by GBS and distributed across the genome to clearly identify the species boundary. Several methods comparing the analyzed strains, from mismatch distributions to PCA to modelbased analyses, consistently identified the same species boundary, even though each species shared over $30 \%$ of its polymorphisms with the other species. Our results also support the presence of only two species in our sample.

Given the clear species boundary between $F$. temperatum and $F$. subglutinans, it remains unclear what, if any, adaptive evolutionary pressure led to their split from a common ancestor and their continued divergence possibly in sympatry. Our study did not find F. temperatum and F. subglutinans co-occurring in any of the five sampled geographic regions, but other studies have found both species in the same region (Moretti et al. 2008; Steenkamp et al. 2002). Such co-occurrences have cast doubt on the role that geographic isolation may have played in the split and divergence of these species. Strains of $F$. temperatum in our analysis came from the higher and drier regions which also grew local maize varieties rather than widespread commercial hybrids common at lower elevations, so both climatic and subtle host-plant differences could have served as drivers of population divergence. Previous work had indicated a possible role for climate in the separation of these species. F. temperatum was more commonly recovered in colder areas and $F$. subglutinans was more common in warmer and drier regions (Moretti et al. 2008; Reynoso et al. 2004), though others have reported that both species are adapted to similar climatic conditions (Czembor et al. 2015). The possibility that adaptation to climate could explain either the speciation event or the current distribution of the species has important implications under predicted climate change scenarios.

Most strains of both species also differ in a species-specific manner in the suite of mycotoxins they produce (Fumero et al. 2020a). This difference suggests that their pathogenic interactions with host plants also may differ and may have been critical in the process of speciation. Modern agricultural practices may have helped blur the relationship between $F$. subglutinans and F. temperatum. A clearer picture of differences may be seen if strains isolated from native plants from noncultivated regions were analyzed rather than those from monoculture crops, and if more strains were analyzed from the Americas, where the species split is thought to have occurred. Finally, experiments measuring growth rates and the ability to infect different host varieties under various environmental conditions could provide further clues about factors important in the separation of the species.

The close relationship between the two species, which can serve as a model for speciation and divergence within the FFSC, is evident in the large number of polymorphisms they share throughout their genomes. Two potential explanations for the shared polymorphisms, a recent split between the two species and gene flow between the species subsequent to their split, are not mutually exclusive. Our population genomic data, sampling more deeply within each species, allows us to test each of these explanations by the demographic modeling of the joint allele frequency spectrum, and provides some support for both.

From a number of models tested to describe the relationship between $F$. temperatum and $F$. subglutinans, an isolation-withmigration model fits the best. Though our GBS loci have some sequence dependence based on the restriction enzyme cut sites, they are nearly randomly scattered across the genome, and include both protein coding and noncoding regions. Due to the dominant action of purifying selection in protein coding regions (Hurst 2009), our estimate of $\theta$, the population mutation rate, must be lower than one estimated strictly from silent nucleotide changes that are presumably neutral. Similarly, our estimates of effective population size that are derived from the estimate of $\theta$ also are likely to be underestimates. The data's largest departure from the model's expectations result from an excess of derived alleles found at low frequency in both species. The action of purifying selection on nonsynonymous mutations in coding regions could produce this excess, since selection can prevent deleterious mutations (i.e., derived alleles) from increasing beyond low frequencies (Nielsen 2005).

The species split time estimation requires several assumptions, including mutation rate and generation time, but our rough estimate

TABLE 4. Range in hermaphrodite frequencies and length for equilibrium cycles based on observed data for Fusarium subglutinans and F. temperatum

\begin{tabular}{|c|c|c|c|c|c|c|c|c|c|c|c|}
\hline \multirow[b]{2}{*}{ Species } & \multicolumn{4}{|c|}{ Time $^{\mathrm{a}}$} & \multicolumn{3}{|c|}{ Hermaphrodite frequency } & \multicolumn{4}{|c|}{ Time $^{\mathrm{b}}$} \\
\hline & $0.90^{\mathrm{c}}$ & $0.95^{\mathrm{c}}$ & $0.98^{\mathrm{c}}$ & $0.99^{\mathrm{c}}$ & Max. $^{d}$ & Obs. & Min. $^{e}$ & $0.90^{\mathrm{c}}$ & $0.95^{\mathrm{c}}$ & $0.98^{\mathrm{c}}$ & $0.99^{\mathrm{c}}$ \\
\hline F. subglutinans $(n=46)$ & 13.0 & 26.6 & 67.6 & 136 & 0.255 & 0.065 & 0.004 & 25.9 & 53.2 & 135 & 272 \\
\hline
\end{tabular}

a Time, in asexual generations, to cycle from $h_{a}$ (frequency of hermaphrodites immediately following the sexual generation) to the observed frequency given the value of $(1-\mu) \Theta$.

b Time, in asexual generations, to cycle from the observed frequency to $h_{b}$ (frequency of hermaphrodites immediately preceding the sexual generation) given the value of $(1-\mu) \Theta$.

c $(1-\mu) \Theta$, where $\mu$ is the mutation rate from female-fertile to female-sterile, and $\Theta$ is the selective disadvantage associated with hermaphroditism during asexual propagation.

d Value for $h_{a}$ if the observed hermaphrodite frequency is used as $f_{s_{0}}$ in equation 3 from Leslie and Klein (1996).

e Value for $f_{s_{O}}$ if the observed hermaphrodite frequency is used as $h_{a}$ in equation 3 from Leslie and Klein (1996). 
is on the order of hundreds of thousands of years ago, clearly before the domestication of the primary agricultural host, maize. This timing is consistent with the 8.8 Mya date for the most recent common ancestor of the FFSC, and is even smaller than the estimated radiation ( 0.8 Mya) of the homothallic Fusarium head blight pathogens within the $F$. graminearum species complex (O’Donnell et al. 2013).

Our analysis also provides strong support for gene flow between the two species subsequent to their split. The direction of gene flow has a clear bias from $F$. subglutinans into F. temperatum, though we do not know enough about the evolution and intermating of these species to know what could have caused this bias. Future analyses with strains collected from other continents could shed light on the role of gene flow during these species' divergence, as these populations should all be subsamples from the original populations in the Americas.

Semipermeable species boundaries, such as those that would allow the genetic exchange required to explain our data, can affect the population genetics and evolution of the species and have important implications for pathogen control. The semipermeability of species boundaries in fungi, though not well appreciated until recently, is not limited to Fusarium species and is currently reflected by the not-infrequent reports of phenomena such as cross-fertility and hybrid species, horizontal gene transfer, and introgressive hybridization (Harrison and Larson 2014; Kohn 2005; Leslie et al. 2004a, b; Steenkamp et al. 2018). In other population genomic studies, a history of relatively high gene flow between diverging populations or species has been demonstrated for fungal populations of Coccidioides and Neurospora (Ellison et al. 2011; Neafsey et al. 2010).

The presence and relative frequency of sexual and clonal reproduction vary greatly across Fusarium species and even within populations. Population genomic studies like ours allow inferences on the mode of reproduction. A history of genetic recombination, and hence sexual reproduction, is clear given the observed decay of LD and inferred recombination events in each species and the inference of historical gene flow between the two species. Yet the presence of clusters of closely related strains in each species also reflects asexual reproduction that is ongoing and neither infrequent nor insignificant.

The largest cluster in each species in our study represents over $25 \%$ of the strains from that species. Approximating each cluster as a single haplotype, haplotype diversity is slightly lower in $F$. temperatum than in $F$. subglutinans. This relatively lower diversity and slower LD decay in F. temperatum both support the hypothesis of less historical recombination in this species. This pattern is expected if clonal reproduction is relatively more frequent. More frequent clonal reproduction also could explain the strong skew observed in the MAT allele ratio in F. temperatum. The fraction of female-fertile strains, which is low in both species, also can provide information on the relative frequency of sexual reproduction in recent times. Although this fraction is slightly lower in F. subglutinans, it is not significantly different from that in $F$. temperatum, so recent rates of sexual reproduction appear similarly low for both species. Finally, the historical gene flow indicates occasional interspecific hybrid crosses have occurred and may still be occurring in nature.

The $F$. temperatum and $F$. subglutinans populations from Argentina have similar levels of genetic diversity but differ in ways besides the frequency of sexual reproduction in their past, such as in the evidence for population structure and recent selective sweeps. Our selection scan revealed much greater evidence for sweeps in F. temperatum, again supporting the separation of the two entities

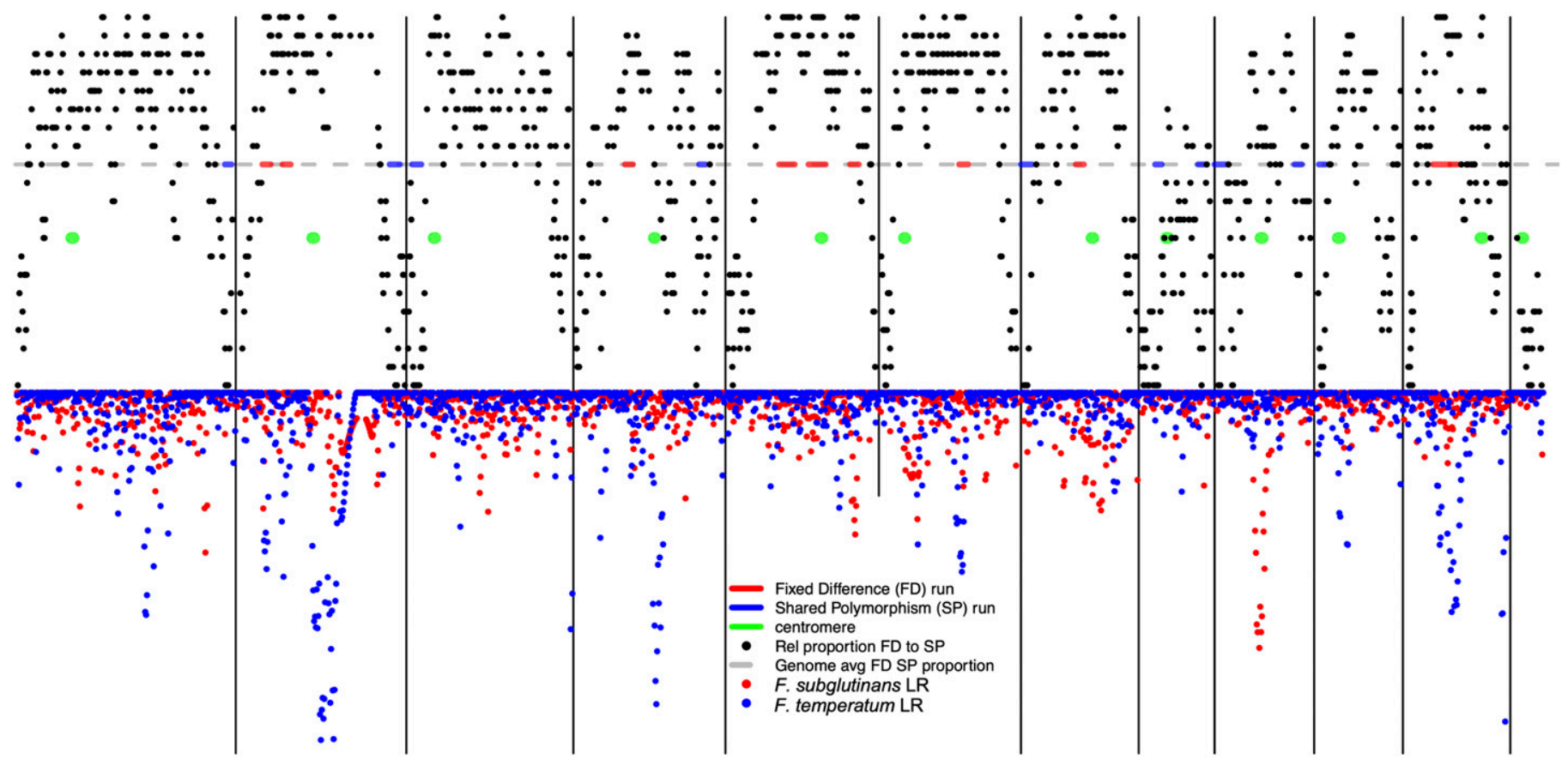

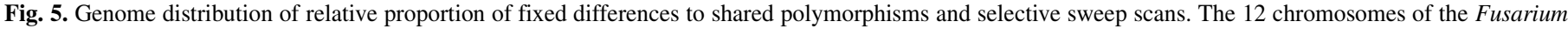

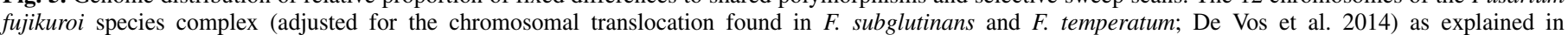

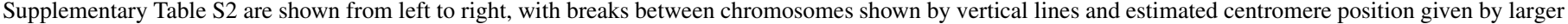

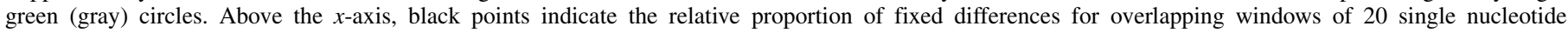
polymorphisms (SNPs) that are either fixed differences or shared polymorphisms (five SNPs offset between each consecutive point). The highest points correspond

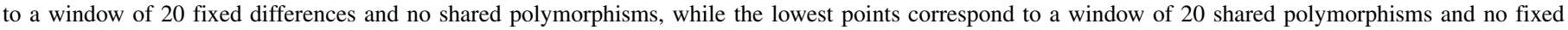

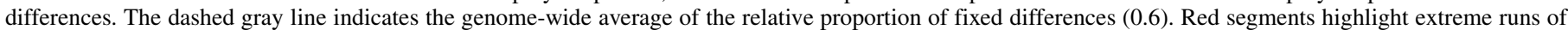

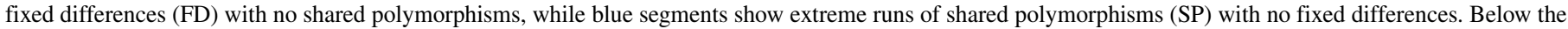

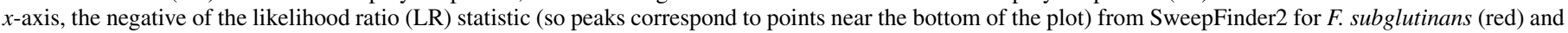

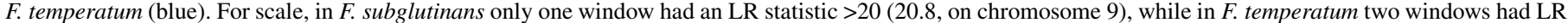
statistics $>28$ (28.2 and 28.3, on chromosome 2). 
into distinct taxa despite their apparently similar environments and phenotypic similarities. However, moderate evidence for a common sweep across both species, possibly facilitated by gene flow between them, suggests that the evolution of these species may not be completely independent.

Inference of population structure within each species is complicated in our study by the small sample sizes and the presence of clusters of closely related strains. When taken as a whole, however, our results support the presence of population subdivision in $F$. temperatum but not in $F$. subglutinans. Any patterns of subdivision in F. subglutinans, such as in the PCA or fineRADstructure results, appear to result solely from the presence of clonal lineages. In contrast, patterns from $F$. temperatum are consistent with population substructure at a level beyond just the clonal lineages. Notably, a comparison of the mismatch distribution for each species indicates that while the maximum level of mismatch in $F$. subglutinans is $17.5 \%$, the mismatch distribution in F. temperatum extends well to the right, up to $27 \%$ with a mode at $25 \%$. The population substructure we observed in $F$. temperatum has no clear relationship to sampling location. The higher background level of LD between unlinked SNPs in F. temperatum may result from substructure in this species, though the smaller sample size from this species also probably contributes to this pattern. Whether these general patterns hold for F. temperatum and F. subglutinans populations outside Argentina remains to be determined.

Sequence-based genome-wide markers enable the measurement of variation at high resolution along chromosomes. This resolution allows for the identification of regions that may be important in maintaining species boundaries or that have been subjected to a recent selective sweep. In this study, the subtelomeric regions at the ends of the chromosomes had higher levels of nucleotide variation and also were enriched for shared polymorphisms. High levels of variation at the ends of chromosomes is a hallmark of the welldocumented genome compartmentalization in other species of Fusarium, such as $F$. graminearum and $F$. fujikuroi. These regions also have higher per nucleotide recombination rates than the rest of the genome (Chiara et al. 2015; Cuomo et al. 2007; Laurent et al. 2017, 2018; Walkowiak et al. 2016).

Subtelomeric regions are enriched in genes that are expressed in planta or encode secreted proteins, which are likely to have a role in host-pathogen interactions or ecological niche adaptation (Chiara et al. 2015; Cuomo et al. 2007; Walkowiak et al. 2016). Most strains of $F$. temperatum can produce the mycotoxin beauvericin, while most strains of $F$. subglutinans cannot, due to mutations in the Beal gene (Fumero et al. 2020a). Beal is located near the end of chromosome 9 where both intraspecific genetic diversity and the relative proportion of shared polymorphisms are higher than average. In contrast, the central regions of chromosomes of both species generally have low levels of variation and are enriched for fixed differences between the species. These central regions are characterized by lower recombination rates and a higher concentration of housekeeping genes, such as $\beta$-tubulin, EF- $1 \alpha$, and RPB2, which have been found to be informative for species identification.

The enrichment in putative host-pathogen interaction genes found near chromosome ends could contribute to the high level of shared polymorphism found there. Pathogen innovations in one species that increase fitness, perhaps in an environment-specific manner, would more likely become established in both species after crossing the semipermeable reproductive barrier separating the species. Yet the nonrandom distribution of observed fixed differences and shared polymorphisms also may be consistent with simpler models. These models might not require genetic adaptation of the pathogens to explain patterns of shared polymorphisms, once factors such as varying recombination rates across the genome and their interaction with selective sweeps and/or background selection are taken into account (Stephan 2010). Whether the subtelomeric enrichment of shared polymorphisms has resulted from a simple neutral explanation, e.g., genomic patterns of recombination, or is related to selection on genes in these genomic regions, will require more extensive future analyses.

In conclusion, our population genomic analysis of strains from $F$. subglutinans and $F$. temperatum has provided strong evidence that these two entities are different species that have a history of genetic exchange with each other. This genetic exchange may still be occurring occasionally in the field, since in the laboratory a low level of interfertility exists between some strains in the different species. Finally, since the genome organization of these species is similar to that already observed in other species of Fusarium, they share the same contrast in genetic variation levels between putative core and adaptive genome regions. Our strains are from Argentina, though both $F$. subglutinans and $F$. temperatum are known to occur worldwide. The relationship between geographically dispersed populations of these species is an important question for future study. In particular, it will be interesting to determine if the clonal lineages detected in the Argentina populations have migrated elsewhere and are prevalent in other populations too. Members of widespread clonal lineages could be of particular interest for study to identify the traits that have contributed to their success. Further population genomic studies with other members of the FFSC should shed further light on the potential for toxin production, pathogenicity, and strain resilience of these species when faced with major climate and environmental changes.

\section{ACKNOWLEDGMENTS}

We thank H. Sarlangue and L. Lanzavecchia from INTA Balcarce and INTA Necochea (Buenos Aires); F. Canteros, O. Figueroa, and E. Moreno from INTA Tucumán; J. Schimpf, H. Farfán, and J. A. de Pasquale from the National University of Jujuy, Argentina for assistance sampling maize. We also thank Alec J. Coffman for help identifying the correct null distribution for the $\delta$ a $\delta$ i likelihood ratio test performed, Vikram Chhatre for help implementing StrAuto, Upasana Dhakal for comments on the manuscript, and Bruce Ramundo and Amy Beyer for laboratory assistance.

\section{LITERATURE CITED}

Afgan, E., Baker, D., van den Beek, M., Blankenberg, D., Bouvier, D., Cech, M., Chilton, J., Clements, D., Coraor, N., Eberhard, C., Grüning, B., Guerler, A., Hillman-Jackson, J., Von Kuster, G., Rasche, E., Soranzo, N., Turaga, N., Taylor, J., Nekrutenko, A., and Goecks, J. 2016. The Galaxy platform for accessible, reproducible and collaborative biomedical analyses: 2016 update. Nucleic Acids Res. 44:W3-W10.

Al-Hatmi, A. M. S., Hagen, F., Menken, S. B. J., Meis, J. F., and de Hoog, G. S. 2016. Global molecular epidemiology and genetic diversity of Fusarium, a significant emerging group of human opportunists from 1958 to 2015. Emerg. Microbes Infect. 5:e124.

Aoki, T., O’Donnell, K., and Geiser, D. M. 2014. Systematics of key phytopathogenic Fusarium species: Current status and future challenges. J. Gen. Plant Pathol. 80:189-201.

Asai, S., Ayukawa, Y., Gan, P., Masuda, S., Komatsu, K., Shirasu, K., and Arie, T. 2019. High-quality draft genome sequence of Fusarium oxysporum f. sp. cubense strain 160527, a causal agent of Panama disease. Microbiol. Resour. Announc. 8:e00654-e19.

Bertazzoni, S., Williams, A. H., Jones, D. A., Syme, R. A., Tan, K. C., and Hane, J. K. 2018. Accessories make the outfit: Accessory chromosomes and other dispensable DNA regions in plant-pathogenic fungi. Mol. PlantMicrobe Interact. 31:779:788.

Cárcamo, M. I., García, M. M., Manzur, M. I., Montoro, Y., Pengue, W., Salgado, A., and Vélez, G. 2011. Biodiversidad, erosión y contaminación genética del maíz nativo en América Latina. Red por una América Latina Libre de Transgénicos. Mas Grafica, Santiago.

Catchen, J., Hohenlohe, P. A., Bassham, S., Amores, A., and Cresko, W. A. 2013. Stacks: An analysis tool set for population genomics. Mol. Ecol. 22: 3124-3140.

Chiara, M., Fanelli, F., Mulè, G., Logrieco, A. F., Pesole, G., Leslie, J. F., Horner, D. S., and Toomajian, C. 2015. Genome sequencing of multiple isolates highlights subtelomeric genomic diversity within Fusarium fujikuroi. Genome Biol. Evol. 7:3062-3069.

Coleman, J. J., Rounsley, S. D., Rodriguez-Carres, M., Kuo, A., Wasmann, C. C., Grimwood, J., Schmutz, J., Taga, M., White, G. J., Zhou, S. G., 
Schwartz, D. C., Freitag, M., Ma, L. J., Danchin, E. G. J., Henrissat, B., Coutinho, P. M., Nelson, D. R., Straney, D., Napoli, C. A., Barker, B. M., Gribskov, M., Rep, M., Kroken, S., Molnar, I., Rensing, C., Kennell, J. C., Zamora, J., Farman, M. L., Selker, E. U., Salamov, A., Shapiro, H., Pangilinan, J., Lindquist, E., Lamers, C., Grigoriev, I. V., Geiser, D. M., Covert, S. F., Temporini, E., and VanEtten, H. D. 2009. The genome of Nectria haematococca: Contribution of supernumerary chromosomes to gene expansion. PLoS Genet. 5:e1000618.

Cuomo, C. A., Güldener, U., Xu, J. R., Trail, F., Turgeon, B. G., Di Pietro, A., Walton, J. D., Ma, L. J., Baker, S. E., Rep, M., Adam, G., Antoniw, J., Baldwin, T., Calvo, S., Chang, Y. L., DeCaprio, D., Gale, L. R., Gnerre, S., Goswami, R. S., Hammond-Kosack, K., Harris, L. J., Hilburn, K., Kennell, J. C., Kroken, S., Magnuson, J. K., Mannhaupt, G., Mauceli, E., Mewes, H. W., Mitterbauer, R., Muehlbauer, G., Münsterkötter, M., Nelson, D., O’Donnell, K., Ouellet, T., Qi, W., Quesneville, H., Roncero, M. I. G., Seong, K. Y., Tetko, I. V., Urban, M., Waalwijk, C., Ward, T. J., Yao, J., Birren, B. W., and Kistler, H. C. 2007. The Fusarium graminearum genome reveals a link between localized polymorphism and pathogen specialization. Science 317:1400-1402.

Czembor, E., Stępień, Ł., and Waśkiewicz, A. 2015. Effect of environmental factors on Fusarium species and associated mycotoxins in maize grain grown in Poland. PLoS One 10:e133644.

De Vos, L., Steenkamp, E. T., Martin, S. H., Santana, Q. C., Fourie, G., van der Merwe, N. A., and Wingfield, B. D. 2014. Genome-wide macrosynteny among Fusarium species in the Gibberella fujikuroi complex revealed by amplified fragment length polymorphisms. PLoS One 9: e114682.

DeGiorgio, M., Huber, C. D., Hubisz, M. J., Hellmann, I., and Nielsen, R. 2016. SweepFinder2: Increased sensitivity, robustness and flexibility. Bioinformatics 32:1895-1897.

Edwards, J., Auer, D., de Alwis, S. K., Summerell, B., Aoki, T., Proctor, R. H., Busman, M., and O'Donnell, K. 2016. Fusarium agapanthi sp. nov., a novel bikaverin and fusarubin-producing leaf and stem spot pathogen of Agapanthus praecox (African lily) from Australia and Italy. Mycologia 108: 981-992.

Ellison, C. E., Hall, C., Kowbel, D., Welch, J., Brem, R. B., Glass, N. L., and Taylor, J. W. 2011. Population genomics and local adaptation in wild isolates of a model microbial eukaryote. PNAS 108:2831-2836.

Evanno, G., Regnaut, S., and Goudet, J. 2005. Detecting the number of clusters of individuals using the software STRUCTURE: A simulation study. Mol. Ecol. 14:2611-2620.

Fumero, M. V., Reynoso, M. M., and Chulze, S. 2015. Fusarium temperatum and Fusarium subglutinans isolated from maize in Argentina. Int. J. Food Microbiol. 199:86-92.

Fumero, M. V., Villani, A., Susca, A., Haidukowski, M., Cimmarusti, M. T., Toomajian, C., Leslie, J. F., Chulze, S. N., and Moretti, A. 2020a. Fumonisin and beauvericin chemotypes and genotypes of the sister species Fusarium subglutinans and Fusarium temperatum. Appl. Environ. Microbiol. 86:e0133-20. doi:10.1128/AEM.00133-20

Fumero, M. V., Yue, W., Chiotta, M. L., Chulze, S. N., Leslie, J. F., and Toomajian, C. 2020b. Data sets and computer code for: Population genomics of Fusarium subglutinans and Fusarium temperatum from Argentina. Kansas State University. https://krex.k-state.edu/dspace/handle/2097/40869

Gardiner, D. M. 2018. Genome sequences of three isolates of Fusarium verticillioides. Microbiol. Resour. Announc. 7:e00918-e18.

Grünwald, N. J., McDonald, B. A., and Milgroom, M. G. 2016. Population genomics of fungal and oomycete pathogens. Annu. Rev. Phytopathol. 54: 323-346.

Guo, L., Zhao, G. Y., Xu, J. R., Kistler, H. C., Gao, L. X., and Ma, L. J. 2016. Compartmentalized gene regulatory network of the pathogenic fungus Fusarium graminearum. New Phytol. 211:527-541.

Guo, L. J., Han, L. J., Yang, L. Y., Zeng, H. C., Fan, D. D., Zhu, Y. B., Feng, Y., Wang, G. F., Peng, C. F., Jiang, X. T., Zhou, D. J., Ni, P. X., Liang, C. C., Liu, L., Wang, J., Mao, C., Fang, X. D., Peng, M., and Huang, J. S. 2014. Genome and transcriptome analysis of the fungal pathogen Fusarium oxysporum f. sp. cubense causing banana vascular wilt disease. PLoS One 9:e95543.

Gutenkunst, R. N., Hernandez, R. D., Williamson, S. H., and Bustamante, C. D. 2009. Inferring the joint demographic history of multiple populations from multidimensional SNP data. PLoS Genet. 5:e1000695.

Harrison, R. G., and Larson, E. L. 2014. Hybridization, introgression, and the nature of species boundaries. J. Hered.: 795-809.

Herron, D. A., Wingfield, M. J., Wingfield, B. D., Rodas, C. A., Marincowitz, S., and Steenkamp, E. T. 2015. Novel taxa in the Fusarium fujikuroi species complex from Pinus spp. Stud. Mycol. 80:131-150.

Hudson, R., and Kaplan, N. 1985. Statistical properties of the number of recombination events in the history of a sample of DNA sequences. Genetics 111:147-164.

Hurst, L. D. 2009. Genetics and the understanding of selection. Nat. Rev. Genet. 10:83-93.
Kelly, A. C., and Ward, T. J. 2018. Population genomics of Fusarium graminearum reveals signatures of divergent evolution within a major cereal pathogen. PLoS One 13:e0194616.

King, R., Brown, N. A., Urban, M., and Hammond-Kosack, K. E. 2018. Intergenome comparison of the Quorn fungus Fusarium venenatum and the closely related plant infecting pathogen Fusarium graminearum. BMC Genomics 19:269.

King, R., Urban, M., and Hammond-Kosack, K. E. 2017. Annotation of Fusarium graminearum (PH-1) version 5.0. Microbiol. Resour. Announc. 5: e01479-e16.

Kohn, L. M. 2005. Mechanisms of fungal speciation. Annu. Rev. Phytopathol. 43:279-308

Kumar, L., Breakspear, A., Kistler, C., Ma, L. J., and Xie, X. H. 2010. Systematic discovery of regulatory motifs in Fusarium graminearum by comparing four Fusarium genomes. BMC Genomics 11:208.

Kurtz, S., Phillippy, A., Delcher, A. L., Smoot, M., Shumway, M., Antonescu, C., and Salzberg, S. L. 2004. Versatile and open software for comparing large genomes. Genome Biol. 5:R12.

Kvas, M., Marasas, W. F. O., Wingfield, B. D., Wingfield, M. J., and Steenkamp, E. T. 2009. Diversity and evolution of Fusarium species in the Gibberella fujikuroi complex. Fungal Divers. 34:1-21.

Laurence, M. H., Summerell, B. A., and Liew, E. C. Y. 2015. Fusarium oxysporum f. sp. canariensis: Evidence for horizontal gene transfer of putative pathogenicity genes. Plant Phytopathol. 64:1068-1075.

Laurent, B., Moinard, M., Spataro, C., Ponts, N., Barreau, C., and Foulongne-Oriol, M. 2017. Landscape of genomic diversity and host adaptation in Fusarium graminearum. BMC Genomics 18:203.

Laurent, B., Palaiokostas, C., Spataro, C., Moinard, M., Zehraoui, E., Houston, R. D., and Foulongne-Oriol, M. 2018. High-resolution mapping of the recombination landscape of the phytopathogen Fusarium graminearum suggests two-speed genome evolution. Mol. Plant Pathol. 19:341-354.

Lawson, D. J., Hellenthal, G., Myers, S., and Falush, D. 2012. Inference of population structure using dense haplotype data. PLoS Genet. 8:e1002453.

Leslie, J. F. 1991. Mating populations in Gibberella-fujikuroi (Fusarium section liseola). Phytopathology 81:1058-1060.

Leslie, J. F., and Klein, K. K. 1996. Female fertility and mating type effects on effective population size and evolution in filamentous fungi. Genetics 144: 557-567.

Leslie, J. F., and Summerell, B. A. 2006. The Fusarium. Laboratory Manual. Blackwell Professional, Ames, IA.

Leslie, J. F., Zeller, K. A., Logrieco, A., Mulè, G., Moretti, A., and Ritieni, A. 2004a. Species diversity of and toxin production by Gibberella fujikuroi species complex strains isolated from native prairie grasses in Kansas. Appl. Environ. Microbiol. 70:2254-2262.

Leslie, J. F., Zeller, K. A., Wohler, M., and Summerell, B. A. 2004b. Interfertility of two mating populations in the Gibberella fujikuroi species complex. Eur. J. Plant Pathol. 110:611-618.

Levic, J., Munaut, F., Scauflaire, J., Stankovic, S., Ivanovic, D., and Krnjaja, V. 2018. Polyphasic approach used for distinguishing Fusarium temperatum from Fusarium subglutinans. J. Agric. Sci. Technol. 21:221-232.

Lysøe, E., Harris, L. J., Walkowiak, S., Subramaniam, R., Divon, H. H., Riiser, E. S., Llorens, C., Gabaldón, T., Kistler, H. C., Jonkers, W., Kolseth, A.-K., Nielsen, K. F., Thrane, U., and Frandsen, R. J. N. 2014. The genome of the generalist plant pathogen Fusarium avenaceum is enriched with genes involved in redox, signaling and secondary metabolism. PLoS One 9: e112703.

Ma, L. J., van der Does, H. C., Borkovich, K. A., Coleman, J. J., Daboussi, M. J., Di Pietro, A., Dufresne, M., Freitag, M., Grabherr, M., Henrissat, B., Houterman, P. M., Kang, S., Shim, W. B., Woloshuk, C., Xie, X. H., Xu, J. R., Antoniw, J., Baker, S. E., Bluhm, B. H., Breakspear, A., Brown, D. W., Butchko, R. A. E., Chapman, S., Coulson, R., Coutinho, P. M., Danchin, E. G. J., Diener, A., Gale, L. R., Gardiner, D. M., Goff, S., Hammond-Kosack, K. E., Hilburn, K., Hua-Van, A., Jonkers, W., Kazan, K., Kodira, C. D., Koehrsen, M., Kumar, L., Lee, Y. H., Li, L. D., Manners, J. M., Miranda-Saavedra, D., Mukherjee, M., Park, G., Park, J., Park, S. Y., Proctor, R. H., Regev, A., Ruiz-Roldan, M. C., Sain, D., Sakthikumar, S., Sykes, S., Schwartz, D. C., Turgeon, B. G., Wapinski, I., Yoder, O., Young, S., Zeng, Q. D., Zhou, S. G., Galagan, J., Cuomo, C. A., Kistler, H. C., and Rep, M. 2010. Comparative genomics reveals mobile pathogenicity chromosomes in Fusarium. Nature 464:367-373.

Malinsky, M., Trucchi, E., Lawson, D. J., and Falush, D. 2018. RADpainter and fineRADstructure: Population inference from RADseq data. Mol. Biol. Evol. 35:1284-1290.

Marasas, W. F. O., Rheeder, J. P., Lamprecht, S. C., Zeller, K. A., and Leslie, J. F. 2001. Fusarium andiyazi sp. nov., a new species from sorghum. Mycologia 93:1203-1210.

Matsuoka, Y., Vigouroux, Y., Goodman, M. M., Sanchez, G. J., Buckler, E., and Doebley, J. 2002. A single domestication for maize shown by multilocus microsatellite genotyping. PNAS 99:6080-6084. 
McKenna, A., Hanna, M., Banks, E., Sivachenko, A., Cibulskis, K., Kernytsky, A., Garimella, K., Altshuler, D., Gabriel, S., Daly, M., and De Pristo, M. A. 2010. The Genome Analysis Toolkit: A MapReduce framework for analyzing next-generation DNA sequencing data. Genome Res. 20:1297-1303.

Miyashita, N. M., and Langley, C. H. 1988. Molecular and phenotypic variation of the white locus region in Drosophila melanogaster. Genetics 120:199-212.

Moretti, A., Logrieco, A., Bottalico, A., Ritieni, A., Randazzo, G., and Corda, P. 1995. Beauvericin production by Fusarium subglutinans from different geographical areas. Mycol. Res. 99:282-286.

Moretti, A., Mulé, G., Ritieni, A., Láday, M., Stubnya, V., Hornok, L., and Logrieco, A. 2008. Cryptic subspecies and beauvericin production by Fusarium subglutinans from Europe. Int. J. Food Microbiol. 127:312-315.

Moussa, T. A. A., Al-Zahrani, H. S., Kadasa, N. M. S., Ahmed, S. A., Sybren de Hoog, G., and Al-Hatmi, A. M. S. 2017. Two new species of the Fusarium fujikuroi species complex isolated from the natural environment. Antonie van Leeuwenhoek 110:819-832.

Myers, S. R., and Griffiths, R. C. 2003. Bounds on the minimum number of recombination events in a sample history. Genetics 163:375-394.

Neafsey, D. E., Barker, B. M., Sharpton, T. J., Stajich, J. E., Park, D. J., Whiston, E., Hung, C. Y., McMahan, C., White, J., Sykes, S., Heiman, D., Young, S., Zeng, Q., Abouelleil, A., Aftuck, L., Bessette, D., Brown, A., FitzGerald, M., Lui, A., Macdonald, J. P., Priest, M., Orbach, M. J., Galgiani, J. N., Kirkland, T. N., Cole, G. T., Birren, B. W., Henn, M. R., Taylor, J. W., and Rounsley, S. D. 2010. Population genomic sequencing of Coccidioides fungi reveals recent hybridization and transposon control. Genome Res. 20:938-946.

Niehaus, E. M., Münsterkötter, M., Proctor, R. H., Brown, D. W., Sharon, A., Idan, Y., Oren-Young, L., Sieber, C. M., Novak, O., Pencik, A., Tarkowska, D., Hromadova, K., Freeman, S., Maymon, M., Elazar, M., Youssef, S. A., El-Shabrawy, E. M., Shalaby, A. B. A., Houterman, P., Brock, N. L., Burkhardt, I., Tsavkelova, E. A., Dickschat, J. S., Galuszka, P., Güldener, U., and Tudzynski, B. 2016a. Comparative "omics" of the Fusarium fujikuroi species complex highlights differences in genetic potential and metabolite synthesis. Genome Biol. Evol. 8:3574-3599.

Niehaus, E. M., Studt, L., von Bargen, K. W., Kummer, W., Humpf, H. U., Reuter, G., and Tudzynski, B. 2016b. Sounds of silence: The beauvericin cluster in Fusarium fujikuroi is controlled by cluster-specific and global regulators mediated by H3K27 modification. Environ. Microbiol. 18:4282-4302.

Nielsen, R. 2005. Molecular signatures of natural selection. Annu. Rev. Genet. 39:197-218

O'Donnell, K., Rooney, A. P., Proctor, R. H., Brown, D. W., McCormick, S. P., Ward, T. J., Frandsen, R. J. N., Lysøe, E., Rehner, S. A., Aoki, T., Robert, V. A. R. G., Crous, P. W., Groenewald, J. Z., Kang, S., and Geiser, D. M. 2013. Phylogenetic analyses of $R P B 1$ and $R P B 2$ support a middle Cretaceous origin for a clade comprising all agriculturally and medically important fusaria. Fungal Genet. Biol. 52:20-31.

O'Donnell, K., Sink, S., Libeskind-Hadas, R., Hulcr, J., Kasson, M. T., Ploetz, R. C., Konkol, J. L., Ploetz, J. N., Carillo, D., Campbell, A., Duncan, R. E., Liyanage, P. N. H., Eskalen, A., Na, F., Geiser, D. M., Bateman, C., Freeman, S., Mendel, Z., Sharon, M., Aoki, T., Cosse, A. A., and Rooney, A. P. 2015. Discordant phylogenies suggest repeated host shifts in the Fusarium-Euwallacea ambrosia beetle mutualism. Fungal Genet. Biol. 82:277-290.

Patterson, N., Price, A. L., and Reich, D. 2006. Population structure and Eigen analysis. PLoS Genet. 2:e190.

Perkins, D. D. 1994. How should the infertility of interspecies crosses be designated? Mycologia 86:758-761.

Piganeau, G., and Eyre-Walker, A. 2004. A reanalysis of the indirect evidence for recombination in human mitochondrial DNA. Heredity 92:282-288.

Poland, J. A., Brown, P. J., Sorrells, M. E., and Jannink, J. L. 2012. Development of a high-density genetic map for barley and wheat using a novel two-enzyme genotype by sequencing approach. PLoS One 7:e32253.

Pritchard, J. K., Stephens, M., and Donnelly, P. 2000. Inference of population structure using multilocus genotype data. Genetics 155:945-959.

Purcell, S., Neale, B., Todd-Brown, K., Thomas, L., Ferreira, M. A. R., Bender, D., Maller, J., Sklar, P., de Bakker, P. I. W., Daly, M. J., and Sham, P. C. 2007. PLINK: A tool set for whole-genome association and population-based linkage analyses. Am. J. Hum. Genet. 81:559-575.

Reynoso, M. M., Torres, A. M., and Chulze, S. N. 2004. Fusaproliferin, beauvericin and fumonisin production by different mating populations among the Gibberella fujikuroi complex isolated from maize. Mycol. Res. 108:154-160.

Scauflaire, J., Gourgue, M., and Munaut, F. 2011. Fusarium temperatum sp. nov. from maize, an emergent species closely related to Fusarium subglutinans. Mycologia 103:586-597.

Schaeffer, S. W., and Miller, E. L. 1993. Estimates of linkage disequilibrium and the recombination parameter determined from segregating nucleotide sites in the alcohol dehydrogenase region of Drosophila pseudoobscura. Genetics 135:541-552.

Seo, S., Pokhrel, A., and Coleman, J. J. 2020. The genome sequence of five genotypes of Fusarium oxysporum f. sp. vasinfectum: A resource for studies on Fusarium wilt of cotton. Mol. Plant-Microbe Interact. 33:138-140.
Sokal, J., and Rohlf, R. 1995. Page 887 in: Biometry, 3rd Ed. WH Freeman and Company, New York.

Sperschneider, J., Gardiner, D. M., Thatcher, L. F., Lyons, R., Singh, K. B., Manners, J. M., and Taylor, J. M. 2015. Genome-wide analysis in three Fusarium pathogens identifies rapidly evolving chromosomes and genes associated with pathogenicity. Genome Biol. Evol. 7:1613-1627.

Srivastava, A. K., Kashyap, P. L., Chakdar, H., Kumar, M., Srivastava, A. K., Yadav, J., Jamali, H., Srivastava, R., Sharma, A., Tiwari, P., Singh, A., and Saxena, A. K. 2018. First de novo draft genome sequence of the pathogenic fungus Fusarium udum F02845, associated with pigeonpea (Cajanus cajan L. Millspaugh) wilt. Microbiol. Resour. Announc. 7:e01001-18.

Srivastava, S. K., Huang, X. Q., Brar, H. K., Fakhoury, A. M., Bluhm, B. H., and Bhattacharyya, M. K. 2014. The genome sequence of the fungal pathogen Fusarium virguliforme that causes sudden death syndrome in soybean. PLoS One 9:e81832.

Steenkamp, E. T., Wingfield, B. D., Coutinho, T. A., Zeller, V., Wingfield, M. J., Marasas, W. F. O., and Leslie, J. F. 2000. PCR-based identification of MAT-1 and MAT-2 in the Gibberella fujikuroi species complex. Appl. Environ. Microbiol. 66:4378-4382.

Steenkamp, E. T., Wingfield, B. D., Desjardins, A. E., Marasas, W. F., and Wingfield, M. J. 2002. Cryptic speciation in Fusarium subglutinans. Mycologia 94:1032-1043.

Steenkamp, E. T., Wingfield, M. J., McTaggart, A. R., and Wingfield, B. D. 2018. Fungal species and their boundaries matter-Definitions, mechanisms and practical implications. Fungal Biol. Rev. 32:104-116.

Stephan, W. 2010. Genetic hitchhiking versus background selection: The controversy and its implications. Philos. Trans. R. Soc. B 365:1245-1253.

Talas, F., and McDonald, B. A. 2015. Genome-wide analysis of Fusarium graminearum field populations reveals hotspots of recombination. BMC Genomics 16:996.

Tralamazza, S. M., Rocha, L. O., Oggenfuss, U., Correa, B., and Croll, D. 2019. Complex evolutionary origins of specialized metabolite gene cluster diversity among the plant pathogenic fungi of the Fusarium graminearum species complex. Genome Biol. Evol. 11:3106-3122.

Villani, A., Proctor, R. H., Kim, H. S., Brown, D. W., Logrieco, A. F., Amatulli, M. T., Moretti, A., and Susca, A. 2019. Variation in secondary metabolite production potential in the Fusarium incarnatum-equiseti species complex revealed by comparative analysis of 13 genomes. BMC Genomics 20:314.

Walkowiak, S., Bonner, C. T., Wang, L., Blackwell, B., Rowland, O., and Subramaniam, R. 2015. Intraspecies interaction of Fusarium graminearum contributes to reduced toxin production and virulence. Mol. Plant-Microbe Interact. 28:1256-1267.

Walkowiak, S., Rowland, O., Rodrigue, N., and Subramaniam, R. 2016. Whole genome sequencing and comparative genomics of closely related Fusarium head blight fungi: Fusarium graminearum, F. meridionale and F. asiaticum. BMC Genomics 17:1014.

Weir, B. S., and Cockerham, C. C. 1984. Estimating F-statistics for the analysis of population structure. Evolution 38:1358-1370.

Wiemann, P., Sieber, C. M., Von Bargen, K. W., Studt, L., Niehaus, E. M., Espino, J. J., Hub, K., Michielse, C. B., Albermann, S., Wagner, D., Bergner, S. V., Connolly, L. R., Fischer, A., Reuter, G., Kleigrewe, K., Bald, T., Wingfield, B. D., Ophir, R., Freeman, S., Hippler, M., Smith, K. M., Brown, D. W., Proctor, R. H., Münsterkötter, M., Freitag, M., Humpf, H. U., Güldener, U., and Tudzynski, B. 2013. Deciphering the cryptic genome: genome-wide analyses of the rice pathogen Fusarium fujikuroi reveal complex regulation of secondary metabolism and novel metabolites. PLoS Pathog 9:e1003475.

Wingfield, B. D., Barnes, I., De Beer, Z. W., De Vos, L., Duong, T. A., Kanzi, A. M., Naidoo, K., Nguyen, H. D. T., Santana, Q. C., Sayari, M., Seifert, K. A., Steenkamp, E. T., Trollip, C., Van der Merwe, N. A., Van der Nest, M. A., Wilken, P. M., and Wingfield, M. J. 2015. IMA Genome-F 5: Draft genome sequences of Ceratocystis eucalypticola, Chrysoporthe cubensis, C. deuterocubensis, Davidsoniella virescens, Fusarium temperatum, Graphilbum fragrans, Penicillium nordicum, and Thielaviopsis musarum. IMA Fungus 6:493-506.

Wingfield, B. D., Liu, M., Nguyen, H. D. T., Lane, F. A., Morgan, S. W., De Vos, L., Wilken, P. M., Duong, T. A., Aylward, J., Coetzee, M. P. A., Dadej, K., De Beer, Z. W., Findlay, W., Havenga, M., Kolařík, M., Menzies, J., Naidoo, K., Pochopski, O., Shoukouhi, P., Santana, Q. C., Seifert, K. A., Soal, N., Steenkamp, E. T., Tatham, C. T., van der Nest, M. A., and Wingfield, M. J. 2018. Nine draft genome sequences of Claviceps purpurea s.lat., including $C$. arundinis, $C$. humidiphila, and $C$. cf. spartinae, pseudomolecules for the pitch canker pathogen Fusarium circinatum, draft genome of Davidsoniella eucalypti, Grosmannia galeiformis, Quambalaria eucalypti, and Teratosphaeria destructans. IMA Fungus 9:401-418.

Zhang, Y., and Ma, L. J. 2017. Deciphering pathogenicity of Fusarium oxysporum from a phylogenomics perspective. Pages 179-209 in: Fungal Phylogenetics and Phylogenomics. J. P. Townsend and Z. Wang, eds. Elsevier Academic Press, San Diego. 